\title{
13 Urheberrechts-Wissensgesellschafts-Gesetz (UrhWissG)
}

Am 30. Juni wurde das UrhWissG im Bundestag in 2. und 3. Lesung verabschiedet. Das Gesetz ist zum 1. März 2018 in Kraft getreten. ${ }^{476}$ Sozusagen in letzter Minute wurde nach einer Empfehlung des Rechtsausschusses ${ }^{477}$ beschlossen, die Geltung der neuen Paragraphen auf fünf Jahre zu begrenzen. ${ }^{478}$ Nach vier Jahren soll eine Evaluierung der Auswirkungen der Reform stattfinden (entsprechend §142). Das Gesetz wurde gegen die Stimmen der LINKEN und mit Enthaltung der GRÜNEN beschlossen. Beide Oppositionsparteien hatten bis zum RegE das Vorhaben mitgetragen, wenn auch mit vielen kritischen Einwänden. Die letzten Änderungen, wie so oft durch Intervention des Rechtsausschusses, waren dann zu viel für sie. Die folgende Darstellung ${ }^{479}$ konzentriert sich auf den UrhWissG-Kern mit den $\S \S 60 \mathrm{a}-60 \mathrm{~h}$.

UrhWissG wird von der Politik als die umfassende Reform eines Urheberrechts für Bildung und Wissenschaft angesehen. Tatsächlich ist es eine Korrektur der im Ersten und Zweiten Korb eingeführten Normen anzusehen und nicht als ein grundlegend neuer Ansatz für ein zeitgemäßes Wissenschaftsurheberrecht. Verschiedene Regelungen aus früheren Reformen, die sich nicht als geeignet für ein

476 Neben den acht neuen $\S \S$ (der Kern des UrhWissG) enthält das UrhWissG noch weitere Unterabschnitte. Unterabschnitt 1: Gesetzlich erlaubte Nutzungen; Unterabschnitt 2: Vergütung der nach den $\S \S 53,60$ a bis 60f erlaubten Vervielfältigungen; Unterabschnitt 3: Weitere gesetzlich erlaubte Nutzungen; Unterabschnitt 5: Besondere gesetzlich erlaubte Nutzungen verwaister Werke; Unterabschnitt 6: Gemeinsame Vorschriften für gesetzlich erlaubte Nutzungen.

Dazu wird in Artikel 2 eine „Änderung des Gesetzes über die Deutsche Nationalbibliothek“ vorgenommen und in Artikel 3 eine Änderung des Patentgesetzes.

Zusätzlich einschlägig für Bildung und Wissenschaft ist in Unterabschnitt 1 die Erweiterung des Zitatrechts ( $\$ 51$ UrhG) über einen neuen Satz 3, durch den die Zitaterlaubnis auch für „eine Abbildung und sonstigen Vervielfältigungen des zitierten Werks“ gegeben wird. Das ist für die Praxis der Nutzung hilfreich.

477 Rechtsausschuss 28.6.2017 - https://bit.ly/32cTQ4T.

478 Die fünf Jahre sollen nach der Erläuterung im Schlussantrag für das UrhWissG auch dafür genutzt werden, eine zentrale Online-Lizenzierungsplattform aufzubauen (Bundestagsdruck 18/13014, S. 5 - https://bit.ly/2FOS5AX). Die in §60g festgeschriebene Priorität von gesetzlichen Schrankenregelungen gegenüber Lizenzvereinbarungen ist also keineswegs dauerhaft gesichert. Die Befristung, sofern sie nicht aufgehoben wird, hätte zur Folge, dass die $\S \S 60 \mathrm{a}-60 \mathrm{~h}$ zum 1 . März 2023 aufgehoben werden (entsprechend § 142 Abs. 2 UrhWissG).

479 Der Text in diesem Abschnitt beruht weitgehend auf Überlegungen und Formulierungen aus (Kuhlen 2017b) UrhWissG - das neue Wissenschaftsurheberrecht bleibt regulierungstechnisch überspezifisch problematisch.

Ә Open Access. (C) 2020 Rainer Kuhlen (c))BY This work is licensed under a Creative Commons Attribution 4.0 License. https://doi.org/10.1515/9783110693447-013 
Wissenschaftsurheberrecht herausgestellt hatten, sind nicht reformiert bzw. nicht gelöscht worden, z. B. die Regelung für verwaiste Werke (Abschnitt 11.1) und die für das Zweitverwertungsrecht (Abschnitt 11.3). Bis zum Ende der im Bundestag beschlossenen Befristung von UrhWissG bis 2023 ist keine größere Urheberrechtsreform in Deutschland zu erwarten. Allerdings müssen einige Normen im deutschen UrhG an die 2019 verabschiedete neue EU-Urheberrechts-Richtlinie angepasst werden. ${ }^{480}$

\subsection{Zur politischen Entwicklung des UrhWissG}

Die Bundesregierung ließ sich nach der bis dahin letzten Reform 2013 und nach dem Versprechen im Koalitionsvertrag der Großen Koalition zwischen CDU/CSU und SPD von 2013, eine ABWS in das UrhG einzuführen, viel Zeit, um einen entsprechenden Vorschlag vorzulegen. Die öffentliche Bekanntgabe des im BMJV schon 2017 erarbeiteten RefE wurde immer wieder verzögert - hauptsächlich dadurch, dass wegen des entsprechenden Drucks aus der CDU/CSU bzw. des Drucks der Verleger/des Börsenvereins auf diese erst eine Lösung für eine Beteiligung der Verlage an den Ausschüttungen der Verwertungsgesellschaften gefunden werden sollte. Darauf wurde in Abschnitt 8.7 ausführlich eingegangen. Im Februar 2017 wurde der RefE für das UrhWissG veröffentlicht. Dieser spricht im Titel vom „Entwurf eines Gesetzes zur Angleichung des Urheberrechts an die aktuellen Erfordernisse der Wissensgesellschaft (Urheberrechts-Wissensgesellschafts-Gesetz - UrhWissG)“. An die Stelle der bisherigen primär auf Bildung und Wissenschaft bezogenen Schrankenregelungen (vor allem die §§ 52a, 52b und Teile von 53 und 53a) sollte unter der Überschrift „,Gesetzlich erlaubte Nutzungen für Unterricht, Wissenschaft und Institutionen“ eine ganze Serie von acht neuen Normen $\$ \S 60 \mathrm{a}-60 \mathrm{~h}$ treten. Der zuständige Justizminister, Heiko Maas, hatte schon 2015 die Vorgaben skizziert:481

480 Für eine kritische Analyse von EU-DSM-RL2019 vgl. (Dreier 2019a) Die Schlacht ist geschlagen - ein Überblick. Zum Ergebnis des Copyright Package der EU-Kommission. Tatsächlich geht die „Schlacht“ weiter. Schon Mitte Januar 2020 hat die Bundesregierung begonnen, Umsetzungsvorschläge für einige, auch wissenschaftsrelevante Artikel aus EU DSM-RL vorzulegen, z. B. DMS-RL Art. 12 Kollektive Lizenzvergabe mit Blick auf Verlegerbeteiligung in § 63a und § 27 VGG; DMS-RL Art. 15 (auch mit Berücksichtigung von DMS-RL Art. 3-7) mit Blick auf das Leistungsschutzrecht für Presseverleger in den $\S \S 87 \mathrm{f}-\mathrm{h}$ - https://bit.ly/39rcaLT. Im Juni 2020 hat das BMJV einen zweiten Vorschlag zur Umsetzung von DSM-RL zur Kommentierung durch die Fachöffentlichkeit vorgelegt, z. B. zur Anpassung von Vergütungsregelungen; zu Nicht verfügbaren Werken (Ersatz für vergriffene Werke); Vervielfältigungen gemeinfreier visueller Werke; Umsetzung von Art. 17 (Regelung für Upload-Filter/Plattformen) - https://bit.ly/2WFOmhM.

481 (Maas 2016) Kulturelle Werke - mehr als nur ein Wirtschaftsgut. 
Es geht dabei zum einen darum, die sehr komplexen Schrankenregelungen des Urheberrechtsgesetzes für Bildung und Wissenschaft neu zu ordnen und verständlicher zu fassen unverständlicher geht auch einfach nicht mehr. Dies wird auf Basis des geltenden Unionsrechts geschehen, denn auf die angekündigten Vorschläge aus Brüssel und deren Umsetzung auf EU-Ebene wollen wir dann doch nicht warten.

Die Lösung der Probleme mit dem bestehenden Wissenschaftsurheberrecht sollte dadurch geschehen, dass a) die bestehenden Schranken „neu geordnet, konsolidiert und vereinfacht“ werden, b) „die Erlaubnistatbestände [für die Nutzungshandlungen - RK] soweit geboten und nach derzeitigem Unionsrecht zulässig“ erweitert werden und c) ,gesetzlich erlaubte Nutzungen [sind] regelmäßig angemessen zu vergüten" seien.

Das BMJV hatte mit der Veröffentlichung seines Vorschlags zugleich die davon betroffenen Kreise aufgefordert, zu dem Entwurf Stellung zu nehmen. Die bis zum 29. Mai 2017 eingegangenen (weit über hundert) Stellungnahmen wurden erfreulicher Weise (bis dahin in der Politik nicht immer üblich) öffentlich zugänglich gemacht. ${ }^{482}$ Trotz aller Vielfalt und entgegen vereinzelten totalen Ablehnungen in den Stellungnahmen sah doch die Mehrzahl der Kommentare im UrhWissG-RefE einen weiteren Schritt in die richtige Richtung, auch wenn das immer mit einigem Bedauern verbunden war. So erinnerte Thomas Hoeren daran, dass vorgesehen war,

eine Generalschranke in weiter Formulierung und Anlehnung an das amerikanische FairUse-Prinzip ins Urheberrecht einzuführen. Diese Bitte blieb aber unerhört. Der Gesetzgeber beharrte auf dem Prinzip der starren, enumerativen Schranken. ${ }^{483}$

Die Opposition im Bundestag - z. B. Kai Gehring, Sprecher von Bündnis90/Die Grünen - maß ebenfalls den RefE an dem Versprechen der Koalition, eine Bildungsund Wissenschaftsschranke einzuführen: „Der jüngst bekannt gewordene Referentenentwurf zum Wissenschaftsurheberrecht enthält jedoch keine solche allgemeine Schrankenregelung. Das widerspricht der Vereinbarung im Koalitionsvertrag. “484 Er bezweifelt, ob die Koalition ,in der Lage ist, das Urheberrecht für die Belange der Bildung und Wissenschaft zukunftsfest und innovationsfreundlich zu gestalten.“ Ähnlich so die Einschätzung von Seiten der LINKE.

Die Bundesregierung (das Bundeskabinett) hatte am 12. April 2017 diesen Gesetzentwurf für eine Reform des Urheberrechts beraten und mit kleineren Änderungen beschlossen. Anschließend beriet der Bundesrat über diese Version und gab

482 Auf der Website des BMJV zum Gesetzgebungsverfahren des UrhWissG: https://bit.ly/2Ff3dFk.

483 (Hoeren 2018) Das Urheberrechts-Wissensgesellschafts-Gesetz.

484 Kai Gehring/Bündnis90/Die Grünen 18.1.2017 - https://bit.ly/379Kzfb. 
am 12.5.2017 Empfehlungen dazu ab. ${ }^{485}$ In Kap. 8 zur Vergütung haben wir schon erwähnt, dass in diesen Empfehlungen des Bundesrats der Bundesregierung auch nahegelegt wurde, die Vergütungsverpflichtung von schrankenbasierter Nutzung für Lehr- und Lernzwecke in Frage zu stellen. Der Bundesrat bedauerte vor allem, dass die Bundesregierung nicht die „,von den Ländern präferierte Generalklausel“ realisiert hat, akzeptierte aber dann doch den Vorschlag als „akzeptablen Weg für die dringend notwendige Neujustierung der gemeinsam von Bund und Ländern verfolgten wissenschafts- und kulturpolitischen Zielsetzungen im Bereich des Urheberrechts“. In der raschen Antwort der Bundesregierung auf die kritischen Vorschläge des Bundesrates zum UrhWissG bekräftigte die Bundesregierung ihr Ziel,

Nutzungserlaubnisse im Interesse von Bildung und Wissenschaft neu zu ordnen und teilweise zu erweitern, zugleich aber die damit einhergehenden Eingriffe in das Urheberrecht mit angemessener Vergütung zu kompensieren, um so einen angemessenen Interessenausgleich herzustellen. ${ }^{486}$

Sie lehnte aber die konkreten Vorschläge des Bundesrats mit dem Hinweis auf verbindliche unionsrechtliche Vorgaben ab. Der Bundesrat hat dann am 8. Juli 2017 das Gesetz passieren lassen, ohne den Vermittlungsausschuss anzurufen (zustimmungspflichtig sind Urheberrechtsgesetze für den Bundesrat nicht - Urheberrecht ist Bundessache). Ein letzter Versuch, so kurz vor Ende der Legislaturperiode das Gesetz noch zu ändern, erschien wohl als aussichtslos und hätte das Gesetz als Ganzes möglicherweise gefährdet. Das schien nicht im Interesse der Länder zu liegen. Der RegE wurde ohne Änderungen in der ersten Lesung im Bundestag wenige Tage später am 19. Mai 2017 verabschiedet. Dieser RegE wurde im Rechtsausschuss in einer öffentlichen Anhörung am 29. Juni 2017 diskutiert ${ }^{487}$ und mit einer Empfehlung an die Bundesregierung für die 2. und 3. Lesung zurückgegeben. Durch den Rechtsausschuss wurden gegenüber dem RefE, aber auch gegenüber dem RegE weitere Änderungen bzw. Einschränkungen der Nutzung vorgenommen:

485 Bundesrat Drucksache 312/17 vom 12.5.2017 - https://bit.ly/2ugJYFn. Er setzte sich vor allem dafür ein, ,im weiteren Gesetzgebungsverfahren durch eine geeignete Ergänzung in §60f UrhG-E Museen eine gesetzliche Erlaubnis einzuräumen, ihre Bestände über das Internet öffentlich zugänglich zu machen, um dadurch einer breiten Öffentlichkeit besseren Zugang zu urheberrechtlich geschützten Kulturgütern im Bestand der Museen zu ermöglichen.“ (die Bundesregierung lehnte das mit Verweis auf das Unionsrecht ab). Der Bundesrat bedauert es weiter, dass es nicht gelungen sei, „ein stringentes Vergütungssystem für die einzelnen Schrankentatbestände zu entwickeln, eine verlässliche Rechtsgrundlage für die Leihe elektronischer Medien ... zu schaffen und keine Überprüfung der Schrankenfestigkeit von Regimen der Digitalen Rechteverwaltung erfolgt ist.“

486 Gegenäußerung des Justizministeriums vom 17. Mai 2017 Drucksache 18/12378 vom 17.05.2017. Quelle initiative urheberrecht vom 19.5.2017: UrhWissG: Regierung weist Vorschläge des Bundesrats zurück - https://bit.ly/35Qvr5F.

487 Die Stellungnahme der Experten ist dokumentiert auf der Website des Deutschen Bundestags unter: https://bit.ly/2Z7Ovhi; die des Verfassers auch unter: https://bit.ly/2xW5QK7. 
Im Gesetz über die Deutsche Nationalbibliothek (Art. 1, §16a Abs. 2) wurde den Presseverlegern ein exklusives Recht auf die Vermarktung ihrer Archive zugesichert. Ein Eingriff in dieses Recht über das Web-Harvesting der Nationalbibliothek war allerdings nie vorgesehen und nie vorgenommen worden. Nur solche Texte dürfen „geerntet“" werden, die frei zugänglich sind bzw. solche nicht, für welche die langfristige Verfügbarkeit, z. B. durch Pressearchive, gesichert ist. Zudem verständigten sich kurz vor der Abstimmung die Fraktionen der in der Regierung vertretenen Parteien nach intensivem Lobbying der Presse (initiiert durch die FAZ ${ }^{488}$ ) neben der Befristung des UrhWissG auf fünf Jahre noch auf eine weitere Einschränkung, nämlich Zeitungsartikel von den Schrankenbestimmungen ( $\S 60 \mathrm{a}, \mathrm{b}, \mathrm{c}, \mathrm{e}, \mathrm{f}$ ) und vom Web-Harvesting durch die Nationalbibliothek auszunehmen. Danach nahm die Unterstützung des Reformvorhabens durch die meisten beteiligten Akteure (außerhalb der weiteren Verlagswelt) und durch die Oppositionsparteien bis zur endgültigen Verabschiedung erheblich ab. $\mathrm{Zu}$ viele Zugeständnisse hatten die Wissenschaftsverlage bzw. deren Vertretung durch den Börsenverein und die Pressemedien der Politik wie schon so oft erfolgreich abringen können.

\subsection{Die Schrankenregelungen im Einzelnen}

\subsection{1 §60a Unterricht und Lehre}

In den ersten beiden Absätzen von §60a wird geregelt, welche urheberrechtsrelevante Nutzung, in welchem Umfang, für wen und für welchen Zweck privilegiert ist. Es wird nun auch im Gesetz bzw. in dessen Erläuterung klargestellt, was Veranschaulichung des Unterrichts (nicht mehr „im“) bedeutet:

Die Veranschaulichung kann „im“ Unterricht erfolgen, aber auch davor oder danach. Daher erfasst die Vorschrift zum einen auch die Vor- und Nachbereitung der Unterrichtsstunden und zum anderen auch die Prüfungsaufgaben und Prüfungsleistungen, die im Verlauf und zum Abschluss des Unterrichts erstellt werden, sowie die Vor- und Nachbereitung von Prüfungen.

Das war ein kleiner Sieg der teleologischen Auslegung des Gesetzes, welcher allerdings schon der BGH beim Urteil zu §52a zugunsten des „des“ anstelle des „im“ gefolgt war. Auch der Fernunterricht (E-Learning) wird explizit begünstigt. Vorgenommene Vervielfältigungen in dem vorgesehenen Umfang dürfen für die öffentliche Wiedergabe und öffentliche Zugänglichmachung genutzt werden. Klargestellt wird auch, dass Begünstigte nicht nur die Lehrenden und Prüfer, sondern

488 Vgl. dazu die Pressemitteilung des Aktionsbündnis 05/19 vom 22.5.2017 - https://bit.ly/ 2MxbqvS. 
auch die Teilnehmer an der jeweiligen Veranstaltung sind. Das ist im Sinne des didaktisch gewünschten selbstbestimmten Lernens zu begrüßen. Die „Schüler“ (Oberbegriff für alle Lernenden) dürfen die bereitgestellten Materialien nicht an Teilnehmer nachfolgender Jahrgänge weitergeben. Ein weiterer Versuch wird unternommen abzuklären, was unter „nicht kommerziell“ zu verstehen ist. Dafür sei nicht der institutionelle Bezug ausschlaggebend - entsprechend werden auch Privatschulen durch $\S 60$ a begünstigt -, sondern allein, ob der Unterricht darauf ausgerichtet ist, „Gewinn zu erzielen“ oder nicht. Ob diese Klärung in der Praxis ausreicht, muss sich erst noch zeigen. EU-RL-2019 Art. 5 macht deutlich, dass allein entscheidend ist, ob die Verwendung der Materialien für den Unterricht erfolgt. ${ }^{489}$

Auf die Verwendung unbestimmter Rechtsbegriffe wie „kleine Teile“ wird weitgehend verzichtet. Von größeren Werken dürfen bis zu $15 \%$ genutzt (vervielfältigt, verbreitet und öffentlich zugänglich gemacht werden). Im RefE waren zunächst $25 \%$ vorgesehen und auch vom Bundesrat gefordert; vom BGH waren $201312 \%$ als angemessen angesehen worden. Für die Nutzung von Abbildungen, Beiträgen aus Fachzeitschriften, Zeitschriften sowie für „sonstige Werke geringen Umfangs“ gibt es keine Umfangsbeschränkung. Eine großzügige Interpretation könnte es nahelegen, dass damit auch Beiträge in Konferenzproceedings und anderen Sammelbänden gemeint sind. Schwierig ist eindeutig festzulegen, was in $\S 60 \mathrm{a}$ mit „öffentlich“ gemeint ist. Sicher nicht, wie man umgangssprachlich vermuten würde, die allgemeine Öffentlichkeit. Das UrhG bestimmt „öffentlich“ in $\S 12$ und in $\S 15$ (regelt öffentliche Nutzung) unterschiedlich. Das UrhWissG lehnt sich an die Definition von $\S 15$ an. Danach „,handelt es sich bereits um Öffentlichkeit, wenn nur wenige nicht miteinander verbundene Personen das Werk wahrnehmen können.“ Oder anders: als „öffentlich“ gilt, solange die Nutzer eines Werks nicht durch eine persönliche Beziehung miteinander verbunden sind. Daher ist die Nutzung durch Lehrende, Prüfer und Lernende als öffentliche Zugänglichmachung erlaubt allerdings nur für diese Gruppen, nicht für die Allgemeinheit bzw. die allgemeine Öffentlichkeit. Etwas überraschend werden in der Erläuterung zu §60a Nutzungen für „Schulklassen und andere kleine, regelmäßig zusammen unterrichtete Gruppe” als nicht öffentlich angesehen, so dass diese Nutzungshandlungen nicht als „urheberrechtlich relevant“" angesehen werden. Lehrer dürfen daher z. B. Filme in der Gänze zeigen, aber nicht den Film in der Gänze vervielfältigen. Vervielfältigungen

489 EG 20 in EU-DSM-RL2019 bezieht die Schranke auf "all educational establishments recognised by a Member State, including those involved in primary, secondary, vocational and higher education. It should apply only to the extent that the uses are justified by the non-commercial purpose of the particular teaching activity. The organisational structure and the means of funding of an educational establishment should not be the decisive factors in determining whether the activity is non-commercial in nature". 
(gemeint ist Kopieren) dürfen nur bis zu $15 \%$ des Films gemacht und entsprechend auch nur 15\% in der Schulklasse gezeigt werden. Auch dürfen Filme, die im Prinzip ja jetzt unter den Bedingungen der Absätze 1 und 2 genutzt werden können, nicht aus einer Vorführung im Kino oder anderen Live-Veranstaltungen „mitgeschnitten oder live gestreamt werden.“

Die Nutzung von ganzen Zeitungsartikeln für Unterricht und Lehre in §60a Abs. 2 bzw. für Wissenschaftliche Forschung in §60c Abs. 3 - entsprechend auch in den $\S \S 60 \mathrm{~b}$, e und $\mathrm{f}$ - ist von der urheberrechtlichen Privilegierung ausgenommen. Erlaubt ist durch $\S 60 a$ Abs. 1 und $\S 60$ c Abs. 1 die Nutzung auch von Zeitungsartikeln nur in einem (völlig unbrauchbarem) Umfang von $15 \% .490$ Eine strikte Bereichsausnahme gibt es (wie auch schon in dem bisherigen $\S 52 a$ ) für Werke, die ,ausschließlich für den Unterricht an Schulen geeignet, bestimmt und entsprechend gekennzeichnet“ sind (Abs. 3, 3). Hierdurch soll der Primärmarkt für Schulbuchverlage weiter gesichert werden. Daher muss jeder Lehrende bzw. seine Institution bei dem jeweiligen Rechtsinhaber (oft sind es mehrere) um Erlaubnis nachsuchen. Da dies in der Praxis nicht realistisch ist, einigten sich die Länder und VG WORT in der Vergangenheit auf einen Gesamtvertrag ${ }^{491}$ für erlaubte Nutzungen in Schulen in dem Umfang, wie es §52a vorsieht (also „kleine Teile“ etc.). Von Verlegerseite wurde die Forderung gestellt, dass es auch eine Bereichsausnahme für die Nutzung von Lehrbüchern an Hochschulen geben müsse. Das ist in §60a nicht vorgesehen, da die Nutzungssituation dort grundsätzlich eine andere sei als sie im föderalen System an Schulen und bei Lehrbüchern auch in der Forschung wahrgenommen wird.

An der Vergütungsverpflichtung für die Nutzungen entsprechend den gesetzlichen Regelungen in §60a und § 60c wurde nicht gerüttelt, obgleich der Bundesrat (und auch die EU) angeregt hatte, noch einmal zu überprüfen, ob nicht ,weitergehende Ausnahmen von der Vergütungspflicht statuiert werden können“ (vgl. die einschlägige Diskussion in 8.5.4). Diese Überprüfung wäre auch deshalb sinnvoll gewesen, weil es durch EU-DSM-RL2019, EG 24 den Mitgliedsländern freigestellt wird, ob für die ausbildungsbezogenen Nutzungen Vergütungen an die Rechtsinhaber erfolgen sollen oder nicht. Eine Überprüfung bzw. Änderung wird allerdings wohl erst nach der vorgesehenen Evaluierung von UrhWissG 2021 oder 22 möglich sein. Vielleicht, so die Hoffnung und Erwartung, haben sich bis dahin Leitideen weiter in Richtung freier, auch im Sinne von vergütungsfreier Nutzung in Bildung und Wissenschaft entwickelt.

$490 \mathrm{Zu}$ einer Lösung dieses Problems durch einen Gesamtvertrag für Lehre vgl. FN 526 und 527. 491 Gesamtvertrag zu 52a (Schulen) 27.2.2014 - https://bit.ly/2MxtTsm. Für die Zeit von August 2013 bis Juli 2017 zahlten die Länder an die VG WORT pauschal Euro 2.240.000. 


\subsection{2 §60b Unterrichts- und Lehrmedien}

$\S 60 \mathrm{~b}$ Unterrichts- und Lehrmedien ist eine eher ungewöhnliche, bislang im UrhG nicht vorgesehene Schrankenregelung. Es besteht für das BMJV offenbar ein öffentliches Interesse daran, Hersteller von Unterrichts- und Lehrmedien dergestalt zu begünstigen, dass sie in ihre Produkte „zur Veranschaulichung des Unterrichts und der Lehre an Bildungseinrichtungen (§60a) zu nicht-kommerziellen Zwecken“ Teile von urheberrechtsgeschützten Werken genehmigungsfrei in ihre Sammlungen einbinden dürfen. Mit Sammlungen sind Werke gemeint, „die Werke einer größeren Anzahl von Urhebern vereinigen“. Von größeren Werken dürfen nur Teile von bis zu $10 \%$ aufgenommen werden; Werke kleineren Umfangs dürfen, wie in $\S 60 \mathrm{a}$ vorgesehen, in der Gänze dafür genutzt werden (aber keine Zeitungsartikel). Abweichend von anderen Schrankenregelungen dürfen Vergütungen nicht pauschal, sondern müssen genau nach der aktuellen Verwendung abgerechnet werden.

\subsection{3 $§ 60 c$ Wissenschaftliche Forschung}

§60c Wissenschaftliche Forschung ist als Folgeregelung (zusammen mit §60a) für den dadurch zu streichenden §52a UrhG anzusehen. Klargestellt wird, dass sich jedermann, der wissenschaftliche Forschung betreibt, sich auf diese Regelung berufen kann, also auch „unabhängige Forscher“, „Privatgelehrte“. Das ist eine wichtige Klarstellung. Anders als § 52a-Alt verzichtet $\S 60 c$ auf unbestimmte Rechtsbegriffe. Auch die von $\S 52$ a übernommene Formulierung in Abs. 1, 1 ,bestimmt abgegrenzten Kreis von Personen für deren eigene wissenschaftliche Forschung“ dürfte durch die Erläuterung geklärt sein: „Unerheblich ist, ob alle Personen an derselben Einrichtung tätig sind. Auch innerhalb loser Forschungsverbünde dürfen Materialien genutzt werden.“

Für die nach §60c Abs. 1 erlaubten Nutzungshandlungen der Vervielfältigung, Verbreitung und öffentliche Zugänglichmachung dürfen nun bis zu $15 \%$ (im RefE noch $25 \%$ ) eines Werks genehmigungsfrei, aber vergütungspflichtig genutzt werden. Abbildungen, einzelne Beiträge aus derselben Fachzeitschrift oder wissenschaftlichen Zeitschrift, sonstige Werke geringen Umfangs und vergriffene Werke dürfen in der Gänze genutzt werden - aber nicht Artikel aus Zeitungen (vgl. dazu Abschnitt 13.2.1). Vermutlich erlaubt sind auch Werke aus Proceedings und anderen Sammelbänden, obgleich dies nicht explizit erwähnt wird; aber die Formulierung „sonstige Werke geringen Umfangs“ legt dies nahe. Die mediale Gestalt der Werke ist nicht auf Text beschränkt. Die Nutzungserlaubnis von $\S 60$ c Abs. 1 gilt demnach jetzt auch (so wird es in der Begründung festgestellt) für „Filmwerke und grafische Aufzeichnungen von Werken der Musik [...] um die Erforschung solcher Werke zu 
erleichtern. Eine Beeinträchtigung der normalen Verwertung solcher Werke ist dadurch nicht zu befürchten.“

\subsection{4§60d Text und Data Mining}

Die neu aufgenommene „Vorschrift [zum TDM] ermöglicht es, auf gesetzlicher Grundlage Werke mit Inhalten aller Art automatisiert auszuwerten, z. B. Werke mit Texten, Daten, Bildern, Tönen oder audiovisuellen Inhalten, um damit nichtkommerzielle wissenschaftliche Forschung zu betreiben.“ (Erläuterung zum RegE) $\S 60 d$ als neue TDM-Schranke ist von der deutschen Bundesregierung beschlossen worden, bevor die EU ihre Copyright-Richtlinie verabschiedet hat (so auch schon im UK 2014 und in Frankreich 2016492). Allerdings war auch schon im Entwurf der Kommission von 2016 eine solche neue Schrankenregelung vorgesehen. Da die Regelung insbesondere für Wissenschaft der zentrale Beitrag des UrhWissG ist, gehen wir in 13.4 darauf gesondert ein.

\subsection{5 §60e Bibliotheken}

$\S 60 \mathrm{e}^{493}$ begünstigt nur Handlungen von Bibliotheken, die nicht kommerziellen Zwecken dienen. Bislang war nicht ausdrücklich legitimiert, dass digitale Vervielfältigungen der urheberrechtsgeschützten Materialien für „Zwecke der Zugänglichmachung, Indexierung, Katalogisierung, Erhaltung und Restaurierung erstellt werden dürfen“. Das ist jetzt, wie schon durch Gerichtsentscheidungen für richtig befunden, gesetzlich geregelt (Abs. 1). Solche Vervielfältigungen dürfen auch an andere Bibliotheken für Zwecke der Restaurierung weitergegeben werden (Abs. 2). An andere Bibliotheken dürfen ,restaurierte Werke sowie Vervielfältigungsstücke von Zeitungen, vergriffenen oder zerstörten Werken aus ihrem Bestand“ verliehen werden. Auch die Verbreitung solcher Werke in „öffentlicher Ausstellung oder zur Dokumentation des Bestandes der Bibliothek“ ist erlaubt (Abs. 3).

Die für die Nutzung entscheidende öffentliche Zugänglichmachung von digitalisierten Werken aus ihrem Bestand dürfen Bibliotheken weiterhin nur ,an Terminals in ihren Räumen“ für Nutzer zu Forschungszwecken oder privaten Studien ermöglichen (Abs. 4). Die „Leseplatzschranke“ aus §52b-Alt bleibt also weiter

492 Text and Data Mining (TDM) exceptions in the UK and France - https://bit.ly/2kmcyGl.

493 Eine Zusammenfassung der Regelungen durch $\S 60$ e aus Bibliothekssicht durch den dbv https://bit.ly/2KOPU3I; vgl. auch (Schüller-Zwierlein/Leiwesmeyer 2018) Neuerungen im Urheberrecht. Stand und Perspektiven. 
im Gesetz. Von größeren Werken darf jeder Nutzer pro Sitzung (einmal am Tag?) $10 \%$ für den eigenen Gebrauch vervielfältigen, d. h. z. B. auf einen Stick speichern oder ausdrucken. ${ }^{494}$ Annexhandlungen sind auch schon vom BGH für den alten $\S 52 \mathrm{~b}$ für erlaubt angesehen worden; diese sind jetzt auch durch das Gesetz in dem jeweils beschränkten Umfang legalisiert.

Eine 10\%-Beschränkung gilt auch für den Versand auf Bestellung für nichtkommerzielle Zwecke (Abs. 5). Für kleinere Werke, z. B. Artikel aus Fachzeitschriften (keine Kioskzeitschriften), gelten keine Beschränkungen. Artikel aus Zeitungen sind ausgeklammert (entsprechend den Regelungen in §60a und c; vgl. Abschnitte 13.2.1 und 13.2.3). Der Versand ist jetzt (anders als noch in §53a) „technologieneutral und ohne Vorrang von Verlagsangeboten“ erlaubt (so in der Erläuterung). Die alte Beschränkung, dass Artikel nur als grafische Datei bzw. per Fax versandt werden dürfen, ist somit weggefallen. Von ganzen Werken dürfen nur $10 \%$ versandt werden. Bibliotheken dürfen jetzt nicht mehr an Benutzer in kommerziellen Einrichtungen liefern (Abs. 5). Die für Zwecke des TDM erstellten Korpora dürfen die Bibliotheken dauerhaft speichern, um die Überprüfbarkeit der Ergebnisse zu sichern. Die Wissenschaftler selbst müssen diese Daten nach Fertigstellung der TDM-Analysen löschen

Vergütung muss nicht für bibliotheksinterne Dienste/Vorgänge entrichtet werden. Für sonstige schrankenbasierte Nutzungen wird über die Verwertungsgesellschaften pauschal abgerechnet. Die Einzelmeldepflicht, z. B. bezüglich der elektronischen Semesterapparate, scheint, entsprechend $\S 60 \mathrm{~h}$, bis auf weiteres vom Tisch zu sein. Dass Vergütung auch im Kontext der Regelungen durch UrhWissG weiter als unabdingbar angesehen wird und dafür auch individuelle Vergütungsformen angewendet werden, zeigt sich z. B. darin, dass für schrankenbasierte Nutzungen 2019 ein neuer Rahmenvertrag zwischen Bund und Ländern und den Verwertungsgesellschaften VG WORT und VG Bild-Kunst vereinbart wurde. ${ }^{495}$ Dort heißt es in $\S 4$ :

Als angemessene Vergütung für eine öffentliche Zugänglichmachung nach §1 Absatz 1 Satz 1 und die Vervielfältigungen durch die Einrichtungen nach §2 Abs. 2 entrichten die Einrichtungen einmalig pro zugänglich gemachtem Werk an die VG WORT eine Vergütung in Höhe von $120 \%$ des Nettoladenpreises des jeweiligen Schriftwerkes. [...] Wenn Einrichtungen zusätzlich zur öffentlichen Zugänglichmachung auch Vervielfältigungen nach §2 Abs. 2 ermöglichen, ist eine weitere Vergütung in Höhe von $20 \%$ der in Absatz 1 genannten Ver-

494 Gegen diese Regelung richtete sich besonders stark die Verlagsindustrie, da nach zehn jeweils für sich erlaubten Nutzungen das ganze Werk kopiert sein würde.

495 Rahmenvertrag zur Vergütung von Ansprüchen nach §60e Abs. 4 i. V. m. §60h Abs. 1 UrhG von 2019- https://bit.ly/30obB0t. 
gütung zu entrichten. Diese Vergütung ist für jedes Jahr erneut zu bezahlen, in dem die Vervielfältigungen ermöglicht werden.

Das sind schon starke Zugeständnisse an die Interessen der Rechteinhaber (i.d. R. der Verlage). Zum einen müssen Bibliotheken für die Erstellung von Digitalisaten von Werken, die sie in analoger Form schon in ihren Beständen haben, und für deren öffentliche Zugänglichmachung an Terminals in den Bibliotheken $120 \%$ des Nettoladenpreises des jeweiligen Schriftwerks an die VG WORT entrichten. Zum anderen muss $\S 4 \mathrm{Abs}$. 2 wohl so verstanden werden, dass für jede tatsächliche Vervielfältigungshandung (Ausdrucken oder Speichern z. B. auf einen USB-Stick) eine Vergütung von $24 \%$ des Nettoladenpreises gezahlt werden muss - obgleich „je Sitzung“ (§60e Abs. 4 Satz 2) Speichern und Ausdrucken nur von $10 \%$ des gesamten Werks erlaubt ist. Ergänzt wird dieser Rahmenvertrag durch einen weiteren Vertrag zur Regelung des bibliothekarischen Leihverkehrs. ${ }^{496}$ Nach §4 Vergütung sollen 1,87 Euro (+ Umsatzsteuer) für jede ausgelieferte Bestellung an die VG WORT entrichtet werden. Auch hier gilt die Beschränkung des technischen (i. d. R. elektronischen) Versands von selbständigen Werken aus dem Bestand der Lieferbibliothek von $10 \%$. Zeitschriftenartikel dürfen vollständig versandt werden.

\subsection{6 $§ 60$ f Archive, Museen und Bildungseinrichtungen}

Alle Bestimmungen aus $\S 60$ e, bis auf die für den Versand, gelten auch für $\S 60 f$ Abs. 4 verbessert die Zugänglichmachung digitalisierter oder originär elektronischer geschützter Werke. Allerdings ist die elektronische Nutzung auch hier auf die internen Lesegeräte beschränkt.

\subsection{7 $§ 60 \mathrm{~g}$ Gesetzlich erlaubte Nutzung und vertragliche Nutzungsbefugnis}

Im ersten Absatz von $\S 60 \mathrm{~g}$ wird der gesetzlichen Regelung in den Schrankenbestimmungen Priorität gegenüber vertraglichen Regelungen (Lizenzen) eingeräumt. Nach Absatz 2 gilt diese Priorität jedoch nicht für die öffentliche Zugänglichmachung von digitalisierten Werken aus dem Bestand der unter $\S 60 \mathrm{f}$ angeführten Institutionen (Archive etc.). Das ist Bibliotheken nach §60e erlaubt.

496 Vertrag für Vergütung im bibliothekarischen Leihverkehr 2019 - https://bit.ly/2NvSBrI. 


\subsection{8 $§ 60 \mathrm{~h}$ Angemessene Vergütung der gesetzlich erlaubten Nutzungen}

Für alle Nutzungen in den Regelungen im Unterabschnitt 4 des RefE (also §60a60f) haben die Rechtsinhaber entsprechend $\S 60$ h einen Anspruch auf Vergütung so wie das regelmäßig von der Rechtsprechung reklamiert wird. Ausgenommen von der Vergütungspflicht ist die „öffentliche Wiedergabe für Angehörige von Bildungseinrichtungen und deren Familien“, nicht jedoch für diese die öffentliche Zugänglichmachung (Abs. 2). Für die Erhebung der Nutzung und der Berechnung der angemessenen Vergütung werden Pauschalierungsverfahren bzw. repräsentative Stichproben für ausreichend gehalten. Der Gesetzgeber hat sich wohl der Einschätzung angeschlossen, dass wegen des großen Aufwands für individualisierte Erhebung und Abrechnung Bibliotheken bzw. die ihnen zugeordneten Einrichtungen davon absehen würden, von den Schrankenregelungen Gebrauch zu machen. ${ }^{497}$ Eine solcher Verzicht würde ,jedoch die Gemeinwohlinteressen beeinträchtigen“, die sich u.a. „auf das Ziel guter und umfassender Bildung“ richten (Erläuterung zum UrhWissG). Die Pauschalvergütung gilt nicht für Nutzungen nach $\S 60 \mathrm{~b}$. Auf die TDM-Regulierung wird im nächsten Abschnitt gesondert ausführlich eingegangen.

\subsection{TDM im UrhWissG und in EU-DSM-RL2019}

Text and Data Mining (TDM) - oft vereinfacht als Big Data angesprochen - gehört zu den technologischen, besser methodischen Entwicklungen der letzten Jahre, mit denen sich das (europäische und auch das deutsche) Urheberrecht schwer getan hat - aus systematischen urheberrechtlichen Gründen, aber auch wegen der früheren Entscheidung in der EU, über eine spezielle Datenbank-Richtlinie besondere Rechte und entsprechenden Schutz den Datenbankerstellern zuzubilligen und damit Einschränkungen für die Nutzungen dieser Datenbanken auch in Bildung und Wissenschaft in Kauf zu nehmen. Das sah und sieht z. B. in den USA anders aus, da nach vielen Gerichtsentscheidungen TDM als sogenannte transformative Technologie angesehen wird, deren Anwendung, allerdings von Fall zu Fall, durch das Prinzip des Fair use legitimiert wird - nicht zuletzt auch dadurch, dass in die ursprünglichen geschützten Rechte der Rechtsinhaber nicht (oder nur

497 Dies zeichnete sich auch schon Ende letzten Jahres bei der Weigerung der Hochschulen ab, dem Rahmenvertrag für die Nutzung nach §52a UrhG zwischen KMK und VG WORT beizutreten, der Individualerhebung und- abrechnung vorsah. Diese Weigerung hätte zur Folge gehabt, dass z. B. ein Großteil der digitalen Semesterapparate nicht mehr hätte genutzt werden können. 
unwesentlich) eingegriffen werde bzw. der zugefügte Schaden nur minimal sei. ${ }^{498}$ Diese Argumentation hat sich auch die EU in DSM-RL $2019 \mathrm{zu}$ eigen gemacht.

TDM selber, also die Anwendung von analytischen Methoden, ist ganz offensichtlich nicht urheberrechtlich relevant. ${ }^{499}$ Urheberrechtlich relevant sind aber die Verfahren des Aufbaus von TDM-Korpora. TDM als Data Mining (DM) zielt darauf ab, durch Verwendung von algorithmischen Verfahren große Datenbestände durchzuarbeiten, um daraus neue Erkenntnisse abzuleiten, wie z. B. neue Datenmuster, neue Trends, extrahiertes neues Wissen. Für viele Wissensgebiete wie Medizin, Klimaforschung, Gentechnologie, Astronomie (wie auch in den meisten MINT/STM-Fächern) sind solche Verfahren unabdingbar geworden, da bei ihnen umfängliche Datenmengen laufend generiert werden, die durch maschinenunabhängige, intellektuelle Analysen nicht mehr bearbeitet werden können. Daher besteht ein öffentliches Interesse daran, dass das exklusive Verwertungsrecht der Vervielfältigung durch eine Schrankenregelung eingeschränkt wird. Damit werden Handlungen zum Zwecke des TDM genehmigungsfrei möglich. Aber auch in so gut wie allen anderen Wissenschaftsbereichen entstehen ebenfalls große, überwiegend elektronisch verfügbare Datenmengen, aber auch große Textmengen, die ebenso nur noch mit Hilfe von Algorithmen des Text Mining bearbeitet werden können. In der Linguistik sind z. B. traditionelle Anwendungsbereiche wie automatische Sprechererkennung, automatische Übersetzung oder Wissensrepräsentationssprachen für Künstliche Intelligenz ohne Text Mining nicht mehr denkbar - auch wenn oft dafür andere Bezeichnungen wie automatische Textanalyse, automatische Lernverfahren, Text Knowledge Engineering, Web Content Mining etc. verwendet werden. ${ }^{500}$

TDM ist in vielen elaborierten Ausprägungen nicht durch Streaming möglich das wäre dann urheberrechtlich vermutlich nicht relevant, weil dadurch keine

498 (Cox 2015) Text and Data mining and fair use in the United States verwendet für die in den USA eingeführten TDM-Schranken die gleiche Begründung: Nur unwesentlicher Eingriff in bestehende Recht und minimaler Schaden für Rechtsinhaber.

499 (Hilty/Richter 2017) hatten sich entschieden gegen die Ansicht der EU-Kommission in deren Vorschlag für eine TDM-Schranke von 2016 gewandt ("wrongly suggests“), dass für TDM eine Erlaubnis durch die Rechtsinhaber der Ausgangsmaterialien erforderlich sei: "Especially in the case that a user has lawful access to contents (the user has acquired the relevant data as such or has acquired access to them on a contractual basis), the automated analysis of these contents must be permitted, just as reading by the human being does not require any separate consent by the rightholder.” (in Abschnitt 2) Dem ist dann in Deutschland RegE zu §69d gefolgt: „Die automatisierte Auswertung selbst, der Kern des sogenannten Text und Data Mining, ist keine urheberrechtlich relevante Handlung“; auch die EU vertritt 2019 diese Position.

500 Vgl. (Drees 2016) Text und Data Mining: Herausforderungen und Möglichkeiten für Bibliotheken, S. 49. 
Vervielfältigungshandlungen vorgenommen werden müssen. TDM ist bei den meisten Anwendungen auf das Kopieren bzw. Extrahieren und das Zusammenspiel verschiedener Datenmengen angewiesen. Diese Datenmengen sind überwiegend nicht frei verfügbar, sondern werden von kommerziellen Betreibern aufgebaut und gepflegt. Diese Datenmengen bzw. Teile von ihnen müssen für den Zweck der TDM-Analyse in Korpora übertragen werden, was aus urheberrechtlicher Sicht eine Vervielfältigung darstellt. Vervielfältigung ist für das Urheberrecht ohne Erlaubnis der Rechtsinhaber ein Eingriff in deren Verwertungsrechte. Auch eine einzige Kopie ist schon eine Vervielfältigung. Der Vervielfältigungsschutz gilt nicht für Werke und Datenbestände, für die der urheberrechtliche Schutz wegen abgelaufener Schutzfrist nicht mehr gilt oder die aus welchen Gründen auch immer sich in der Public domain befinden.

Sowohl durch das UrhWissG als auch durch EU-DSM-RL2019 sollen in erster Linie die Rechtsunsicherheiten im Hinblick auf Text und Data Mining beseitigt werden, die für Forscher insbesondere durch die frühere EU-spezifische Datenbankrichtlinie entstehen können. ${ }^{501}$ DSM-RL reguliert aber, anders als bislang die deutsche Lösung in den $\S \S 87 f$ ff, TDM über zwei Artikel - in Art. 3 für Zwecke der wissenschaftlichen Forschung und über Art. 4 allgemeine Ausnahmen und Beschränkungen, vor allem für die kommerzielle Nutzung. ${ }^{502}$ Nach Art. 3 von EU-DSM-RL2019 bleiben kommerzielle Organisationen von der TDM-Schranke ausgeschlossen, aber durch Art. 4 Abs. 1 wird ihnen doch für Zwecke des TDM „Vervielfältigen und Entnahmen“ als Ausnahmen von den Rechten der Rechtsinha-

501 Ausführlich zu TDM in UrhWissG und vor allem zu Art. 3 und 4 der EU-DSM-RL2019 (Raue 2019. Rechtssicherheit für datengestützte Forschung). Die EU schaffe „die dringend erforderliche unionsweite Rechtssicherheit für Text- und Data-Mining in der wissenschaftlichen Forschung. Erfreulich ist, dass der Anwendungsbereich der Schranke durch ausführliche, auslegungsleitende Erwägungsgründe näher ausgeleuchtet wird. Wettbewerbsnachteile für die europäische Datenwirtschaft baut die allgemeine Text-und-Data-Mining-Schranke des Art. 4 von EU-DSM-RL2019 ab, die erst in letzter Minute in die Richtlinie aufgenommen worden ist. Sie ist eine zwingende Schranke des Unionsrechts, die hochwertige Datenanalysen möglich macht, weil sie nicht nur temporäre, sondern auch längerfristige Vervielfältigungen erlaubt. Problematisch ist, dass Rechteinhaber einen Nutzungsvorbehalt erklären und die Schranke vertraglich abbedingen können. Sie war aber der bestmögliche Kompromiss im Gesetzgebungsverfahren, um datengetriebene Forschung nun auch in Unternehmen rechtssicher zu erlauben. Immerhin muss der Nutzungsvorbehalt ausdrücklich und im Regelfall in maschinenlesbarer Form erfolgen.“

502 Entsprechend hat die Bundesregierung Mitte Januar 2020 eine Vorschlag zur Diskussion gestellt, durch den die Vorgaben aus der EU in das deutsche Rechte umgesetzt werden (vgl. FN 480) unter anderem dadurch, dass die Vergütungsverpflichtung für TDM-Nutzungen nicht mehr strikt für erforderlich gehalten wird (vgl. https://bit.ly/39rcaLT). Die Darstellung der deutschen Regelung in diesem Text folgt allerdings dem bislang gültigen Text in $\S 60 d$. Änderungen sind aber durch die Umsetzung von DSM-RL bis Juni 2021 zu erwarten. 
ber der Ausgangsmaterialien erlaubt. Durch Art. 4 soll also die Entwicklung der Datenanalyse und der künstlichen Intelligenz außerhalb der nicht kommerziellen Forschung gefördert werden. Allerdings wird dieses Recht durch Art. 4 Abs. 3 der RL dadurch eingeschränkt, dass es nicht gilt, wenn die Rechtsinhaber von Datenbanken einen „Nutzungsvorbehalt“ ihre „Werke und sonstigen Schutzgegenstände“ mit „maschinenlesbaren Mitteln“ (gemeint ist wohl DRM) geschützt haben. Das BMJV hat die Erweiterungen (und Einschränkungen) der TDM-Regelungen durch die EU in ihrem RefE-Entwurf von 1/2020 (zwangsläufig) weitgehend übernommen. (vgl. FN 502)

$\mathrm{Zu}$ beachten ist, dass die TDM-Erlaubnisse nicht den Zugang zu den geschützten Materialien allgemein legitimieren. Auch eine TDM-Schranke (wie alle Schrankenregelungen) ist kein Freibrief zur „Piraterie“. Wie bei anderen Schranken handelt es sich auch bei Nutzungen für TDM immer um bereits (von den Einrichtungen der Forscher) auf legalem Wege (Kauf oder Lizenz) erworbene Materialien. Sowohl die bisherige deutsche TDM-Norm (§60d) als auch Art. 3 und Art. 4 in EU-DSM-RL2019 machen den rechtmäßigen Zugang zu geschützten Materialien (Art. 3 Abs. 1 und Art. 4 Abs. 1) zur Bedingung für die für TDM erforderlichen Vervielfältigungshandlungen. Wie auch bei anderen Schrankenregelungen können bei der deutschen Regelung die gesetzlich erlaubten Nutzungen auch bei der TDM-Schranke nicht durch vertragliche Regelungen ausgesetzt werden. DSM-RL macht davon allerdings in Art. 4 Ausnahmen.

\subsubsection{TDM-Analysekorpora}

Entscheidend für den Erfolg und die wissenschaftliche Akzeptanz von TDM ist der Aufbau von großen Analysekorpora und die nachhaltige Sicherung des Zugriffs auf diese. Durch $\S 60 d$ Text und Data Mining ist die automatische Vervielfältigung von Daten-/Textmengen (,Ursprungsmaterial“) zum Aufbau von Analysekorpora erlaubt. ${ }^{503}$ Die TDM Erlaubnis bezieht sich auch auf Veränderungen dieses Material zur „Normalisierung, Strukturierung und Kategorisierung“ der Korpora“ (Abs. 1, 1). Ohne dies ist kein TDM möglich. Als positiv ist anzumerken, dass in beiden Regulierungen (in der EU und in Deutschland) für TDM keine Begrenzung für den Umfang der zu kopierenden bzw. extrahierenden Materialien vorgesehen

503 Die deutsche TDM-Schranke ist schon vor EU-DSM-RL2019 als neue Schranke in das UrhG eingefügt worden, obgleich nach der damals (und in Teilen auch heute noch) gültigen EU-InfoSocRL2001 der dort aufgeführte Schrankenkatalog als abschließend bestimmt worden war. Allerdings war dem deutschen Gesetzgeber bekannt, dass die EU eine TDM-Regelung als Schranke einführen wollte. 
ist - also weder eine quantifizierende prozentuale Beschränkung noch eine über unbestimmte Rechtsbegriffe wie kleine Teile etc. Das ist zwar selbstverständlich, da sonst TDM nicht sinnvoll durchgeführt werden könnte; aber es ist doch ein Hinweis auch für andere Schrankenregelungen, in denen solche Beschränkungen häufig vorgenommen werden.

$\S 60$ d übernimmt in Abs. 1, 2 die auf 52a-Alt zurückgehende Formulierung, dass die für Zwecke des TDM erarbeiteten Analysekorpora „einem bestimmt abgegrenzten Kreis von Personen für die gemeinsame wissenschaftliche Forschung “ öffentlich zugänglich gemacht werden können. Ebenso ist für „einzelne Dritte“ die „Überprüfung der Qualität wissenschaftlicher Forschung“ erlaubt. Das kann als das Recht interpretiert werden, ${ }^{504}$ auf die Korpora (nicht auf die Ausgangs-/Rohmaterialien) zum Zweck von Kontrolluntersuchungen zurückgreifen zu dürfen, aber auch zum Zwecke des Reviewing von Arbeiten, die TDM-Ergebnisse enthalten, welche aus diesen Korpora abgeleitet wurden. Beides ist für die Qualität von wissenschaftlichem Arbeiten unabdingbar. Die in FN 504 zitierte Begründung für $\S 60 d$ Abs. 3 Satz 1 verpflichtet jedoch die Wissenschaftler, die für die Korpora erstellten Kopien nach Beendigung der TDM-Arbeit zu löschen und auch die Korpora nicht öffentlich zugänglich zu machen. Durch §60d Abs. 3 Satz 2 wird das Löschen aber dadurch relativiert, dass die Korpora und die Vervielfältigungen des Ursprungsmaterials den Forschungsgruppen zugeordneten Institutionen wie Bibliotheken zur dauerhaften Aufbewahrung übermittelt werden sollen. „Aufbewahrung“ klingt sehr defensiv-archivarisch. Die aufbewahrten Materialien sollten auch für die oben zitierte „Überprüfung der Qualität wissenschaftlicher Forschung“ den dafür berechtigten überprüfenden und bewertenden Wissenschaftlern zugänglich gemacht werden.

In EU-DSM-RL2019 in Art. 3 Abs. 2 wird das deutlich offensiver formuliert: „Vervielfältigungen und Entnahmen von Werken oder sonstigen Schutzgegenständen, die gemäß Absatz 1 angefertigt wurden, sind mit angemessenen Sicherheitsvorkehrungen zu speichern und dürfen zum Zwecke der wissenschaftlichen Forschung, auch zur Überprüfung wissenschaftlicher Erkenntnisse, aufbewahrt werden.“ Das wird nun auch in das deutsche Gesetz bei der vorgesehenen Anpassung an EU CR-RL 2019 übernommen - vermutlich auch die Formulierung in CR-RL2019

504 Dies wird bestätigt durch die Begründung der TDM-Schranke im RegE zu Abs. 3: „Die Forscher wollen und müssen die für ihre Forschung benutzten Inhalte weiterhin in Gänze verfügbar halten, um die Zitierbarkeit, Referenzierbarkeit und die Überprüfung der Einhaltung wissenschaftlicher Standards zu ermöglichen.“ Allerdings heißt es dann weiter: „Andererseits haben gerade die Wissenschaftsverlage ein berechtigtes Interesse daran, dass keine parallelen Artikeldatenbanken entstehen. Der Forscher selbst darf nach Abschluss des Forschungsprojekts das Korpus und das Ursprungsmaterial hingegen nicht mehr aufbewahren: Die Kopien sind zu löschen und die öffentliche Zugänglichmachung ist zu beenden“ (Drucksache 18/12329, 15.6.2017). 
Art. 4 Abs. 2: „Vervielfältigungen und Entnahmen nach Absatz 1 dürfen so lange aufbewahrt werden, wie es für die Zwecke des Textes und Data Mining notwendig ist.“ Letzteres bezieht sich bei der EU allerdings auf TDM außerhalb der nicht kommerziellen Forschung.

\subsubsection{TDM - nicht-kommerziell oder auch kommerziell}

Das TDM-Recht in Deutschland ist nach §60d beschränkt auf die automatisierte Auswertung von Korpora für die wissenschaftliche Forschung in nicht-kommerzieller Absicht (§60d Abs. 1). Das geht konform mit der allgemeinen Rechtfertigung für Schranken, dass die Einschränkung der exklusiven Rechte der Rechtsinhaber nur durch ein öffentliches Interesse gerechtfertigt ist. Die EU sieht das offensichtlich bei ihrer TDM-Regelung entspannter. Das wird schon an der Definition von Forschungsorganisationen deutlich (Art. 2 der RL). Darunter werden Forschungsorganisationen allgemein verstanden, nicht nur Universitäten und oder Ausbildungseinrichtungen, sondern eben Organisationen, die ihre Gewinne in wissenschaftliche Forschung reinvestieren (Art. 2 Abs. 1, a) oder die ihre erzielten Forschungsergebnisse nicht exklusiv ihrer Organisation zur Verfügung stellen (Art. 2 Abs. 1, b). Die EU ist doch stark an dem Nutzen von TDM für Forschungsaktivitäten interessiert, die auf kommerzielle Verwertung abzielen und hat, wie oben erwähnt, entsprechend den auf nicht kommerzielle Forschung abzielenden Art. 3 durch einen Art. 4 ergänzt:

Im Einklang mit der derzeitigen Forschungspolitik der Union, die Hochschulen und Forschungsinstitute zur Zusammenarbeit mit der Privatwirtschaft anhält, sollten auch Forschungsorganisationen eine solche Ausnahme nutzen dürfen, sofern ihre Forschungstätigkeit im Rahmen öffentlich-privater Partnerschaften durchgeführt wird. Forschungsorganisationen und Einrichtungen des Kulturerbes sollten auch künftig zu den Begünstigten der Ausnahmeregelung zählen, sich aber bei der Durchführung des Text und Data Mining auch ihrer privaten Partner bedienen können, einschließlich unter Nutzung ihrer technischen Werkzeuge. (EG 11)

Die Anwendung von TDM für kommerzielle Zwecke steht in diesem Text nicht im Vordergrund, aber der Hinweis sei erlaubt, dass ein durch $\S 60 d$ bestimmter Ausschluss der kommerzielle TDM-Nutzung einen erheblichen Wettbewerbsnachteil Deutschlands gegenüber Ländern wie USA oder Japan nach sich ziehen könnte. In den USA ist TDM durch die Fair-Use-Doktrin sowieso weitgehend gedeckt und wird umfassend genutzt. Auch würde die erforderliche und erwünschte Zusammenarbeit der öffentlich finanzierten bzw. im öffentlichen Interesse liegenden Forschung mit industrieller Forschung zwar nicht ganz ausgeschlossen (erlaubt scheint sie zu sein, wenn die industrielle Forschung nicht direkt kommerziellen Zwecken dient), aber vermutlich doch stark behindert. Eine solche Behinderung wird vom BDI als 
„wirtschafts- und realitätsferne Verengung“ abgelehnt. ${ }^{505}$ Fraglich also, warum rein kommerzielle TDM-Nutzungen nicht auch privilegiert werden sollen, wenn sie nur auf „rechtmäßig zugängliche“ Materialien ausgerichtet sein dürfen. Ein öffentliches Interesse am kommerziellen Einsatz von TDM bzw. der Entwicklung entsprechender Produkte und Dienstleistungen ist ersichtlich. ${ }^{506}$ (Hilty/Richter 2017) (vgl. FN 499) hatten zudem zu Bedenken gegeben, dass durch die Erlaubnis zur kommerziellen TDM-Nutzung sich neue Geschäftsfelder auch für die Rechtsinhaber der Ausgangsmaterialien ergeben können, wenn sie für die TDM-Betreiber Dienstleistungen anböten, z. B. zur Normalisierung der Ausgangsmaterialien oder auch zur Unterstützung der Algorithmen. Auf solche Veränderungen in den Geschäftsmodellen der Akteure auf den Informationsmärkten gehen wir in Kap. 14 ein.

Bislang ist es entsprechend den Vergütungsvorgaben der $\$ \S 54$ bis $54 \mathrm{c}$ erforderlich, für die durch $\S 60 \mathrm{~d}$ erlaubten schrankenbasierten TDM-Nutzungen eine Vergütung zu entrichten. Art 4 der EU-DSM-RL2019 sieht so etwas nicht explizit vor (erst recht nicht über Art. 3). Lizenzen für die Ausgangsmaterialien sind ja schon erworben worden, und der Schaden für die Rechtsinhaber wird von der EU als minimal eingeschätzt. Wie in FN 502 erwähnt, wird sich der deutsche Gesetzgeber vermutlich dieser Einschätzung anschließen.

\subsubsection{TDM im Zusammenhang der Regulierung von Datenbanken}

$\S 60 d$ des deutschen Gesetzes ist nicht unabhängig von den Urheberrechtsregelungen für Datenbanknutzungen als Folge der EU-Richtlinie 96/9/EG über den rechtlichen Schutz von Datenbanken von März 1996 zu sehen. Durch §60d Abs. 2 wird TDM explizit in den Kontext von Schrankenregelungen mit Blick auf Datenbanken gestellt, vor allem über $\S \S 55 \mathrm{a}$ und die $87 \mathrm{~b}$ und $\mathrm{c}$ und damit auch unter Berücksichtigung der DRM-Schranken 95a und 95b (s. unten). §60d ist also zunächst als Ergänzung bzw. teilweise auch als Korrektur von $\S 55 \mathrm{a}$ anzusehen, welcher für sich durch UrhWissG nicht geändert wurde. Nach §55a gab/gibt es nach Satz 3 ein unabdingbares Schranken-/Nutzerrecht, die Datenbank eines Rechtsinhabers zu nutzen - allerdings nicht vollständig, sondern nur in vom Rechtsinhaber erlaubten Teilen. Der Rechtsinhaber darf also dieses Recht beschränken. Aber er kann auch die gesamte Datenbank zur Nutzung freistellen. Der Schutz des Rechtsinhabers

505 BDI (Spitzenorganisation der deutschen Industrie und der industrienahen Dienstleister) https://bit.ly/2NyMYK6.

506 Dazu Future TDM - https://bit.ly/3aahK4Z. Tatsächlich hat sich die Bundesregierung in dem in FN 502 erwähnten ersten Diskussionsvorschlag zur Umsetzung von DSM-RL konsequent entschlossen die DSM-RL Unterscheidung zwischen nicht-kommerzieller und kommerzieller TDM-Nutzung (Art. 3 und 4) aufzugreifen. 
wird auch dadurch verstärkt, dass er sein Datenbankwerk entsprechend §95a mit technischen Schutzmaßnahmen (DRM) sichern kann. Durch die Änderung in $\S 95 \mathrm{~b}$ gehören aber jetzt die §§60a-f zu den Schrankenbestimmungen, durch welche die Rechtsinhaber verpflichtet sind, den dadurch rechtlich Legitimierten die Mittel zu überlassen, durch die die technischen Schutzmaßnahmen außer Kraft gesetzt werden können. Alle diese gesetzlich erlaubten Nutzungshandlungen sind durchweg zeitkritisch. Wenn im Zweifelsfall die Anti-DRM-,Mittel“ nicht, wie von $\S 95 b$ vorgesehen, von den Rechtsinhabern gewährt werden, muss das erst zeitraubend über Gericht geklärt werden - mit dem Ergebnis, dass die Nutzung dann keinen Sinn mehr ergibt. DRM-Verbote/Erlaubnisse sollten insgesamt für das Wissenschaftsurheberrecht nicht zur Anwendung kommen.

$\S 60 d$ wendet zudem die auf Datenbankwerke bezogene semantische Regelung in $\S 55 a$ Satz 1 an, wonach die „Bearbeitung oder Vervielfältigung ... für dessen übliche [kursiv: RK] Benutzung“ erlaubt ist. Auch die für TDM erlaubten Nutzungen sind (entsprechend $\S 60 d$ Abs. 1) jetzt auch ,als übliche Benutzung nach $§ 55 a$ Satz 1“ anzusehen (§60d Abs. 2 Satz 1). Der unbestimmte Rechtsbegriff „üblich“ kann also durchaus zeitgemäß ausgelegt werden. Durch $\S 60 d$ Abs. 2 Satz 2 werden weitere unbestimmte Rechtsbegriffe eingeführt: Wenn z. B. „unwesentliche [kursiv: RK] Teile von Datenbankwerken“ nach Abs. 1 genutzt werden, dann kann das „mit den berechtigten Interessen des Datenbankherstellers im Sinne von § 87b Absatz 1 Satz 2 und 87e als vereinbar“ angesehen werden. Was sind unwesentliche Teile? Auch die Erlaubnis für diese gilt nicht unbeschränkt. Nach § 87b Satz 2 können Nutzungen von Datenbanken insgesamt oder in „unwesentlichen Teilen“ [kursiv: RK] dem Rechtsinhaber vorbehalten bleiben, wenn sie der „normalen Auswertung der Bank [durch den Rechtsinhaber] zuwiderlaufen.“ Was ist „normal“? „Normal“ unterläuft aber „unwesentlich“.

Die TDM-Schranke ist auch im Zusammenhang von § 87c „Schranken des Rechts des Datenbankherstellers“ zu sehen. Auch dort sind jetzt als Folge von UrhWissG die $\S \S 60 \mathrm{c}$ und 60d mit Blick auf wissenschaftliche Forschung und $\S \S 60 \mathrm{a}$ und $60 \mathrm{~b}$ für Unterricht und Lehre als begünstigte Schrankenerlaubnisse aufgeführt. Allerdings gilt das alles nach $\S 87 c$ nur für die ,Vervielfältigung eines nach Art oder Umfang wesentlichen Teils einer Datenbank“. Vielleicht ein kleiner Fortschritt gegenüber dem „Unwesentlichen“. Aber für Zwecke des TDM werden häufig die gesamten Datenbanken benötigt.

\subsubsection{Zusammenfassung, Kritik und Fazit für die TDM-Regelung}

Die deutsche Regelung für TDM in §60d ist inzwischen von EU-RL 2019 eingeholt worden. In der Begründung des RegE in Deutschland ist vorgesehen, dass $§ 60 d$ 
dann „soweit erforderlich angepasst“ wird. Tatsächlich ist die Bundesregierung, wie oben erwähnt, schon dabei, die Umsetzung der EU-Vorgaben aus DSM-RL in die Wege zu leiten. Das sollte bis Juni 2021 abgeschlossen sein. Wie schon erwähnt, wird dadurch vermutlich die bislang durch $\S 60 \mathrm{~h}$ vorgegebene Vergütungsverpflichtung für Nutzungen über die TDM-Schranke $\S 60 \mathrm{~d}$ relativiert bzw. vielleicht ganz aufgegeben. Auch wird die jetzige Ausklammerung der nicht-kommerziellen TDM-Forschung durch die DSM-RL Vorgabe aufgegeben werden. Es sollte in einer neuen deutschen TDM-Regelung deutlicher gemacht werden, in welchen Fällen und unter welchen Bedingungen die Beteiligung kommerzieller Organisationen an Public-private-Verbundprojekten mit der Wissenschaft durch die TDM-Schranke erlaubt sein kann. Weitere kritische Anmerkungen zu den TDM-Regelungen:

Den Unterschieden zwischen Data Mining und Text Mining wird nicht explizit Rechnung getragen. Ebenso wird nicht deutlich genug zwischen TDM für den temporären Zugriff über Streaming, der rein technischen Charakter hat, und der längerfristigen Speicherung von vervielfältigten Materialien für komplexe TDM-Anwendungen unterschieden. Ebenso ist nicht geklärt, für welche Fälle die Erlaubnis der vorübergehenden Vervielfältigungshandlungen zutreffend sein kann.

Es ist umstritten, ob es überhaupt eine Notwendigkeit für eine Schrankenbestimmung für TDM gibt, wenn für TDM ein legaler Zugriff auf die zu analysierenden Materialien schon besteht. Wenn man diese berechtigterweise lesen darf, warum soll man sie nicht auch analysieren dürfen - so die Argumentation - entsprechend der Forderung: ,the right to read is the right to mine." ${ }^{507}$ Die Allianz der deutschen Wissenschaftsorganisationen scheint z. B. den Standpunkt zu vertreten, dass es sich bei TDM insgesamt um eine urheberrechtlich nicht relevante Handlung handelt. ${ }^{508}$ Insgesamt ist umstritten, ob das Urheberrecht durch TDM-Aktivitäten überhaupt betroffen ist. Daten, Fakten, Ideen sind nicht urheberrechtlich geschützt. ${ }^{509}$ Viele der Ausgangsdatenbanken für die TDM-Korpora enthalten tatsächlich Daten, keine Texte (g). Es ist auch problematisch (so Bruch, s. FN 508), dass „mit den jetzt disku-

507 Vor allem in zivilgesellschaftlichen Umgebungen wurde seit langem und sehr häufig die Forderung "the right to read is the right to mine" erhoben; vgl. z. B. Peter Murray-Rust für die Open Knowledge Foundation - https://bit.ly/33YRF67.

508 Vgl. auch Christoph Bruch in WikiBlog 1.12.2017: https://bit.ly/2KP3jZu - bezogen allerdings auf den EU-Kommissions-Entwurf von 2016.

509 Vgl. EDiMA, the European association representing European and global online platforms and other innovative tech companies operating in the EU: „Maintaining that facts and ideas be protected by copyright goes against international legal standards as copyright protection does not extend to factual information about a work. Any copying in the context of TDM is incidental" and does not result in any unreasonably harm to the legitimate interests of the copyright holder -https://bit.ly/2L4cww7. 
tierten und in einigen Jurisdiktionen bereits realisierten engen TDM-Schranken rechtsdogmatisch am Aufbau von Eigentumsrechten an Informationen gearbeitet wird, was sich schon bei der Datenbankschutz-Richtlinie als nicht zielführend herausgestellt hat." Es besteht die Gefahr, dass durch eine neue Schranke implizit ein neues Schutzrecht eingeführt werden könnte.510

Die Rechte der Rechtsinhaber werden auch bei den TDM-Schrankenregelungen in DSM-RL nicht außer Kraft gesetzt: a) Dafür sorgen schon die weiter gültigen Vorgaben des Drei-Stufen-Tests. b) Außerdem bleiben die bisherigen unionsrechtlichen Vorgaben, vor allem durch InfoSoc 2001, weiter auch für TDM gültig (vgl. EG 5 und 6 von EU-DSM-RL2019). c) Auch wird durch EU-DSM-RL2019 geregelt, in welchem Ausmaß Rechtsinhabern erlaubt sein soll, „Entnahmen aus einer Datenbank zu untersagen“ (EG 11) Nach Art 3 Abs. 3 können sie Maßnahmen ergreifen, wenn sie durch starke, z. B. große Datenmengen umfassende TDM-Nutzung die „Sicherheit und Integrität“ ihrer Systeme beeinträchtigt sehen. d) Auch soll z. B. der Schutz technischer Schutzmaßnahmen auch für TDM weiter gelten. Allerdings soll gleichzeitig sichergestellt werden, ,dass durch den Einsatz technischer Maßnahmen die Inanspruchnahme der in dieser Richtlinie festgelegten Ausnahmen und Beschränkungen nicht behindert wird.“(EG 7) Etwas vage soll das dadurch geregelt werden, dass die Rechtsinhaber ,freiwillige Maßnahmen“ bereitstellen, durch die die Nutzung entsprechend der Schrankenregelung nicht behindert wird. Die Aufforderung (in Art. 3 Abs. 4), dass sich Rechtsinhaber und TDM-Anwender über solche Maßnahmen verständigen sollen, würde Forschungsorganisationen in langwierige Verhandlungen verstricken, welche zeitnahe Anwendungen behindern.

Schließlich soll darauf hingewiesen werden, dass eine gesonderte TDM-Schrankenregelung für die nicht-kommerzielle TDM-Forschung weitgehend überflüssig wäre, wenn eine ABWS/K in das UrhG eingefügt würde, durch die auch die Nutzungshandlungen für TDM alleine durch den Zweck von Forschung legitimiert würden. Auch hier sollte gelten, dass die Leistung der Anbieter, z. B. Datenbanken zu erstellen, die für TDM benutzt werden sollen, durchaus vergütet werden sollte, dass aber die TDM-Nutzung selbst vergütungsfrei sein sollte.

510 (Hilty/Richter 2019) Vom Drang, Freiheit zu regulieren sehen die Gefahr eines neuen Schutzrechts, jetzt für Daten, sicherlich auch, aber kommen in ihrer Analyse von EU-DSM-RL2019 zu dem Ergebnis, dass von der Politik in der EU und in Deutschland der Anspruch der Datenwirtschaft auf Dateneigentum richtigerweise abgewehrt wurde (bislang jedenfalls). Vgl. dazu (Hoeren 2019) Datenbesitz statt Dateneigentum. Wichtiger mit Blick auf gesamtgesellschaftliche Vorteile sei, so (Hilty/Richter 2019), allerdings die Frage ,wer zu welchen Zwecken und unter welchen Bedingungen Zugang zu bestimmten Datensätzen erhalten sollte.“ 


\subsection{Zuammenfassung der Kritik am UrhWissG}

In den ersten beiden Abschnitten dieses Kapitels haben wir uns bislang weitgehend auf die Beschreibung der Regelungen im UrhWissG beschränkt. In diesem Abschnitt werden diese kritisch analysiert, zunächst aus der Sicht der betroffenen Akteursgruppen. Von Seiten der Wissenschaftsverlage und der Pressemedien wurde starke Kritik am UrhWissG geübt. Sie sahen nicht nur den Schutz des Urhebers und die Vertragsfreiheit im Rahmen der Privatautonomie durch die Reform stark eingeschränkt, sondern befürchteten als Folge des neuen Gesetzes eine „faktische Enteignung “ der Verlage (so vor allem der Börsenverein ${ }^{511}$ ). Auch einzelne Autoren aus der Wissenschaft wandten sich vehement gegen das neue Gesetz. ${ }^{512}$ Auch für die Verlegerin Barbara Budrich - in ihrer Stellungnahme zum RegE vor dem Rechtsausschuss des Bundestags - stellte „das UrhWissG ... für die Verlage nach geltendem Recht und Gesetz eine entschädigungslose Enteignung dar." ${ }^{513}$

Von Seiten der Wissenschaft schätzte eine große Mehrheit der beim BMJV eingegangenen Stellungnahmen den ursprünglichen RefE des BMJV und auch den ersten RegE als grundsätzlich positiv ein..$^{514}$ Obgleich $\S \S 60 a-60 h$ i. d. R. nicht als Ersatz für eine ABWS angesehen wurde, schätzte die Fachwelt das UrhWissG als wichtigen Schritt in Richtung eines bildungs- und wissenschaftsfreundlichen

511 Börsenblatt 26.5.2017 - https://bit.ly/2TXxZe2.

512 Exemplarisch hierfür der Artikel von Roland Reuss in der NZZ mit der Überschrift „Der Staat legalisiert die Enteignung von Urhebern“, geschrieben nach der Verabschiedung des Gesetzes https://bit.ly/2ulTPus.

513 Stellungnahme zum Regierungsentwurf zum UrhWissG der Verlegerin Barbara Budrich im Rechtsausschuss des Bundestags - https://bit.ly/2UHCg82. Die Ausweitung der Nutzungsbedingungen, z. B. die Erhöhung der Anteile an den zu nutzenden Werken von bislang $12 \%$ auf jetzt $15 \%$ (ursprünglich vorgesehen $25 \%$ ), sei kaum noch mit dem Drei-Stufen-Test vereinbar und bedrohe den Primärmarkt der Verlage. Die in $\S 60 \mathrm{~h}$ präferierte Pauschalabrechnung wird kritisiert; entweder sollte grundsätzlich nach jeder einzelnen Nutzung abgerechnet werden (bevorzugt) oder es sollten angemessene Kompromissmodelle zwischen pauschaler und individueller Vergütung entwickelt werden. Der Vorrang von Schrankenregelungen gegenüber Lizenzangeboten/-vereinbarungen wurde kritisiert. Diese ruiniere die Primärmärkte für alle Informationsprodukte. Vor allem sei eine Bereichsausnahme für Lehrbücher erforderlich, nicht nur für Schulbücher.

514 So z. B. (trotz zahlreicher Änderungsvorschläge) grundsätzlich GRUR; KMK, dbv; Allianz der Wissenschaftsorganisationen; Forschungsgruppe Ethik des Kopierens am Zentrum für interdisziplinäre Forschung (ZiF) in Bielefeld; dbv; DHd - Digital Humanities im deutschsprachigen Raum; DINI; Wikimedia Deutschland; IDS; zahlreiche Universitäten Forschungs- und Wissenschaftsorganisationen (alles einsehbar über die URL in Fußnote 482); Vgl. (Grünberger 2018a) und (Grünberger 2018b) 
Urheberrechts bzw. eines allgemeinen Wissenschaftsurheberrechts ein. ${ }^{515}$ Von den meisten zustimmenden Stellungnahmen wurde anerkannt, dass das vom BMJV zentral verfolgte Ziel der Rechtssicherheit als erreicht anzusehen sei. Viele der Regelungen in den Paragraphen 60a-60h seien überfällig und für Nutzer besser verständlich und seien wohl auch praxisnah und in den Interessen sorgfältig abgewogen. Die ursprünglich vorgesehene Erhöhung der Nutzungserlaubnis wurde von vielen Stellungnahmen als angemessen bezeichnet, die dann beschlossene Reduzierung der Erlaubnis von 25\% auf 15\% allerdings eher als Rückschritt angesehen.

Positiv bewertet wurde die Festschreibung des rechtlichen Vorrangs gegenüber Lizenzangeboten oder -vereinbarungen des Marktes. Auch mit der Präferenz für eine Pauschalabrechnung der schrankenbezogenen Nutzungen sei das UrhWissG den in Forschung und Lehre Arbeitenden und den Auszubildenden entgegengekommen. Die empirischen Daten aus der einschlägigen Osnabrücker Studie (FN 286) und auch die heftig ablehnenden Reaktionen auf den Rahmenvertrag zu $§ 52$ Ende 2016, der eine Einzelabrechnung vorsah, zeigten deutlich, dass eine solche individualisierte Erhebung und Abrechnung keine Akzeptanz in Forschung und Lehre finden kann.

Die tendenziell positive Resonanz aus der Sicht der Wissenschaft kann nicht darüber hinwegtäuschen, dass es im Verlauf des Verfahrens (vom RefE, über den RegE, Rechtsausschuss und Beschluss im Bundestag) zum einen einige Rücknahmen der freieren Nutzungsmöglichkeiten von publiziertem Wissen gab und dass zum anderen vielen weitergehenden Forderungen im UrhWissG nicht entsprochen wurde ganz zu schweigen davon, dass die Ziele der versprochenen ABWS, entgegen der Einschätzung der Regierungsvertreter in der 2. und 3. Lesung im Bundestag - Maas (SPD), Flisek (SPD), Schieder (SPD), Wanka (CDU), Kretschmer (CDU)516 -, nicht erreicht wurden. Die folgende Liste der Defizite ist sehr lang. Sie ist viel zu lang, als dass das UrhWissG als erfolgreiche Reform des Wissenschaftsurheberrechts angesehen werden kann:

(1) Es ist nicht mehr zeitgemäß, dass nach $\S 60$ e Abs. 4 weiterhin nicht von außerhalb auf die digitalisierten Bestände der Bibliotheken zugegriffen werden kann. Weiter gilt, was schon an der entsprechenden Regelung im alten $\S 52 \mathrm{~b}$ kritisiert wurde, dass Benutzer nach wie in die Bibliotheksräume gehen

515 So z. B. (Pflüger/Hinte 2018) Das Urheberrechts-Wissensgesellschafts-Gesetz aus Sicht von Hochschulen und Bibliotheken: „anwenderfreundlicher“, „durch die Angabe von prozentualen Mengenangaben hinsichtlich der zulässigen Weiterverwendung von urheberrechtlich geschützten Materialien ein höheres Maß an Rechtssicherheit“, „die für den Regelfall festgeschriebene Pauschalvergütung [...] als deutlicher Fortschritt gegenüber der bisherigen Rechtslage“.

516 Bundestag Plenarprotokoll18/244 vom 30.6.2017 - https://bit.ly/2k5uODH, Zusatztagesordnungspunkt 13. 
müssen, also keinen legitimen elektronischen Zugriff von wo auch immer (z. B. über VPN) haben. Für Bibliotheken sind die Räume im elektronischen Umfeld aber nicht länger nur physische, sondern auch virtuelle Räume (vgl. 10.1.4). Auch in EU-DSM-RL2019 wird die Beschränkung auf eine exklusive Nutzung in den physischen Räumen der Bibliotheken nicht mehr für zeitgemäß gehalten. Diese Kritik gilt auch für die Nutzung von Museums- und Archivbeständen (s. unten No 10 und 11).

(2) Bei der Definition von Bildungseinrichtungen, die durch §60a privilegiert werden, hätten auch Volkshochschulen oder MOOCs (Massive Open Online Courses) explizit mitberücksichtigt werden sollen. CR-RL2019 definiert Bildungs- bzw. Ausbildungseinrichtungen offener.

(3) Die Bereichsausnahme für Schulbücher Ist in erster Linie ein unzeitgemäßer Heimatschutz für i. d. R. deutsche Schulbuchverlage. Für den deutschen Gesetzgeber bestand dafür keine Vorgabe aus der EU. ${ }^{517}$ Auch EU-DSM-RL2019 schreibt in Art. 5 eine solche Bereichsausnahme nicht zwingend vor. Diese Regelung belegt auch die These, dass Urheberrechtsregelungen häufig die Entwicklung von neuen offenen Dienstleistungen verhindern - wie in diesem Fall für die Entwicklung von Schul“büchern“ bzw. Schulmaterialien nach dem OER-Modell, die kollaborativ erstellt, angepasst fortgeschrieben und offen/frei genutzt werden.

(4) Beiträge in Konferenz-Proceedings und in Sammelbänden jeder Art, sollten explizit (zumindest in den Erläuterungen) in die Nutzungserlaubnisse, z. B. in den §§60a und 60c UrhWissG, einbezogen werden. Solche Beiträge sind in vielen Disziplinen wichtiger als Aufsätze in Zeitschriften. Die Formulierung „sonstige Werke geringen Umfangs“ ist nicht ausreichend bzw. arbeitet wieder mit einem unbestimmten klärungsbedürftigen Rechtsbegriff.

(5) Es ist unklar, ob die aus $\S 53$ UrhG (Privatkopie) Abs. 2, 1 gestrichene Regelung „Vervielfältigungen zum privaten und sonstigen eigenen Gebrauch” und „zum eigenen wissenschaftlichen Gebrauch, wenn und soweit die Vervielfältigung zu diesem Zweck geboten ist und sie keinen gewerblichen Zwecken dient“ jetzt

517 EU-DSM-RL2019, Art. 5 Abs. 2 Verwendet nur "may”: "Member States may provide that the exception or limitation adopted pursuant to paragraph 1 does not apply or does not apply as regards specific uses or types of works or other subject matter, such as material that is primarily intended for the educational market or sheet music, to the extent that suitable licences authorising the acts referred to in paragraph 1 of this Article and covering the needs and specificities of educational establishments are easily available on the market.” Ebenso in EG 23 über „could“: „Member States could, for example, use this mechanism [gemeint ist die Bereichsausnahme für Schulbücher und Noten - RK] to give precedence to licences for material that is primarily intended for the educational market or licences for sheet music.” 
durch §60c übernommen wurde oder sogar erweitert worden ist. ${ }^{518}$ In $\S 53$ war nur von Vervielfältigung die Rede, in §60c auch von Verbreiten und Öffentlich Zugänglichmachung. Zumindest Artikel aus wissenschaftlichen Zeitschriften dürfen vollständig genutzt werden. Das gilt für die eigene wissenschaftliche Forschung. Verbreitet und öffentlich zugänglich gemacht werden darf das für Dritte nur „für einen bestimmt abgegrenzten Kreis von Personen für deren eigene wissenschaftliche Forschung”. Wenn mich ein Wissenschaftler um eine E-Kopie eines Artikels fragt, der in meinem Besitz ist, darf ich die ihm allerdings direkt per Mail schicken, auch wenn ich ihn gar nicht persönlich kenne - eine üblich werdende Praxis in der Wissenschaft.

(6) Auf das pro und contra der TDM-Norm ( $\$ 60 \mathrm{~d}$ ) wurde in Abschnitt 13.3 gesondert eingegangen. Durch die DSM-RL Vorgaben der EU wird deutlich, dass die Bundesregierung ihren bisherigen Vorschlag in $\S 60 \mathrm{~d}$ umarbeiten wird.

(7) Die Nutzungserlaubnisse nach $\S 60$ e Abs. 3 hätten so erweitert werden sollen, dass Bibliotheken zur Bewerbung und Anzeige ihres Bestandes die Vervielfältigungen nicht verbreiten, sondern auch öffentlich zugänglich machen dürfen. Dies hätte auch für Archive etc. in $\S 60 f$ gelten sollen. Auch hier sind Vorgaben aus der InfoSoc 2001-Richtlinie nicht weiter plausibel zu machen.

(8) Leider ist $\S 60 \mathrm{e}$ Abs. 5 so restriktiv gehalten worden, dass der Versand auf Bestellung für Einrichtungen mit kommerziellen Nutzungszwecken nicht erlaubt ist. Die Erlaubnis für eine solche (eng begrenzte und vergütungspflichtige) Nutzung durch die Wirtschaft läge ohne Frage im öffentlichen Interesse.

(9) Museen werden (durch §60f in Verbindung mit §60e) daran gehindert, ihren kulturellen Auftrag über die öffentliche Zugänglichmachung wahrzuneh-

518 Sicherlich kann auch Wissen, das aus der Wissenschaft stammt, „konsumiert“ werden, und das Urheberrecht erlaubt auch (in beschränktem Umfang) Nutzungshandlungen von urheberrechtlich geschützten Werken für den ,privaten und sonstigen eigenen Gebrauch“, „,sofern sie weder unmittelbar noch mittelbar Erwerbszwecken dienen“ ( $\$ 53$ UrhG). Damit wird sozusagen die Privatkopie legitimiert. Allerdings sind nach der zum 1.3.2018 gültig werdenden Urheberrechtsreform die „Erlaubnisse für Vervielfältigungen, die auch die Zwecke Unterricht, wissenschaftliche Forschung und Archivierung betreffen "aus $\S 53$ herausgenommen worden. Nutzungen für wissenschaftliche Zwecke sind daher nicht mehr Teil des Rechts auf Privatkopie. Nutzungen für wissenschaftliche Zwecke sind jetzt in einem neuen §60c geregelt - allerdings, mit Blick auf Vervielfältigung, nun weniger freizügig als dies im alten $\S 53$ der Fall war. Dort hieß es ohne jede weitere Umfangsbeschränkung: „(2) Zulässig ist, einzelne Vervielfältigungsstücke eines Werkes herzustellen oder herstellen zu lassen 1. zum eigenen wissenschaftlichen Gebrauch, wenn und soweit die Vervielfältigung zu diesem Zweck geboten ist und sie keinen gewerblichen Zwecken dient“. In §60c dürfen nur $15 \%$ eines größeren Werkes genutzt werden. Kleinere Werke, wie Artikel aus wissenschaftlichen Zeitschriften, können ganz genutzt werden. Presseartikel sind jetzt ganz aus der genehmigungsfreien Erlaubnis herausgenommen (vgl. 13.2.3). Letzteres war seit 2003 noch bis Ende Februar 2018 erlaubt. 
men. ${ }^{519}$ Für Museen ist lediglich vorgesehen, dass diese körperliche Vervielfältigungsstücke in Zusammenhang mit öffentlichen Ausstellungen oder zur Dokumentation ihrer Bestände verbreiten können. Die gesetzliche Erlaubnis hätte aber auf die öffentliche Zugänglichmachung der elektronischen Vervielfältigungen der geschützten musealen Ausstellungs- und Bestandswerke ausgeweitet werden müssen. Hierfür wäre eine kreative Auslegung der EU-rechtlichen Vorgaben erforderlich gewesen, ebenso für die zeitgemäße Erweiterung der Nutzung der Archivbestände (s. No. 10)

(10) Auch Archive werden (durch $\S 60$ in Verbindung mit $\S 60 \mathrm{e}$ ) daran gehindert, ihren kulturellen Auftrag über die öffentliche Zugänglichmachung wahrzunehmen. Diese Ausklammerung der elektronischen Nutzung von Archiven steht im krassen Widerspruch zum Selbstverständnis der Archivare, die sich eben nicht auf den erwähnten (internen) Primärwert der Sicherung von Dokumenten beschränkt sehen wollen. Archive als nicht nur bewahrende, sondern auch bereitstellende Gedächtnisorganisationen sind, so MenneHaritz, gleichgewichtig dem Sekundärwert verpflichtet, welcher „den Nutzen für die Einsichtnahme in die dabei entstandenen Unterlagen, als den Blick von außen bezeichnet." 520

(11) Leider ist darauf verzichtet worden, eine Öffnungsklausel - entsprechend dem Vorschlag von Schack (FN 455) - im UrhWissG vorzusehen. Öffnungsklauseln sind ein Mittel im Urheberrecht, für bestimmte Nutzungen, vielleicht auch für solche, die zum Zeitpunkt der Gesetzgebung noch unbekannt waren, Ausnahmen für weitergehende Nutzungen zu erlauben. Als Beispiel wäre zu nennen, dass für Zwecke des Reviewing Texte sinnvollerweise dann sogar

519 Die Bundesregierung wies das mit dem Argument zurück, dass „das geltende Unionsrecht Museen nicht gestattet, Werke online zugänglich zu machen.“ Das Gleiche galt für die Bundesregierung für eine erweiterte Öffnung der Archive. Vgl. (Kirchmaier 2017) Das Urheberrechts-Wissensgesellschafts-Gesetz und seine Auswirkungen auf den Bereich der Kultureinrichtungen.

520 (Menne-Haritz 2013) Artikel D 10 Archive. Zusammenfassend die Kritik in der Stellungnahme der Konferenz der Leiterinnen und Leiter der Archivverwaltungen des Bundes und der Länder https://bit.ly/2NpQIOd.

* Regelung einer eigenen Schranken für Archive unter Berücksichtigung der Natur und der Gegenstände des Archivwesens * Onlinepräsentation von Katalogen und Dokumentationen (Findmittel), die geschützte Werke darstellen

* Zugänglichmachung digitaler Werke im Internet (frei oder passwortgeschützt)

* Reproduktionen digitaler Werke von internen Lesegeräten ohne mengenmäßige Begrenzung

* Kopienversand auf Bestellung (ohne mengenmäßige Begrenzung)

* Zugänglichmachen von verwaisten Werken im Internet unter Hinzunahme von Lichtbildern und Lichtbildwerken ohne Beschränkung auf veröffentlichte Werke. 
vergütungsfrei genutzt werden könnten und nicht nur die generell erlaubten $15 \%$ des Werks.

(12) Auf eine Quantifizierung der Nutzungshandlungen (derzeit variierend zwischen 10, 15 und $75 \%$ ) wäre besser ganz verzichtet worden. Die Vermeidung von unbestimmten Rechtsbegriffen (z. B. „kleine Teile“) gaukelt nur Rechtssicherheit vor. Es ist zu erwarten, dass so gut wie niemand sich an Vorgaben wie $15 \%$ halten wird. Entscheidend sollte, analog zu InfoSoc 2001, Art. 5 Abs. 3 lit. a, allein der Zweck der Forschung bzw. der Ausbildungsaktivität sein. ${ }^{521}$

(13) Die Norm für die Nutzung von verwaisten Werken (beschlossen bei der vorletzten Urheberrechtsanpassung von 2014; vgl. 11.1) ist leider nicht korrigiert worden. Diese Vorschrift besagte, dass verwaiste Werke nur dann genutzt (vervielfältigt und öffentlich zugänglich gemacht) werden dürfen, wenn dem eine sorgfältige (höchst aufwendige) Suche (diligent search) vorausgegangen war. Die Bedingung einer solchen Suche erwies sich als Hindernis für die erwünschte Massendigitalisierung für Zwecke digitaler Bibliotheken - auch wenn durch Anwendung der Regelung auf vergriffene Werke diese Restriktion teilweise umgangen werden konnte. ${ }^{522}$ Die Registrierungsregel für vergriffene Werke hätte auch hier zur Anwendung kommen sollen (ausführlicher in 11.2).

(14) Es wurde versäumt, die Regelungen für Vergütung für (meistens öffentlich finanzierte) Werke der Wissenschaft insgesamt und besonders für die Bereiche der Bildung noch einmal grundlegend in Frage zu stellen. Dies hatte der Bundesrat empfohlen (FN 485) und auch die EU für „cross-border education“ nahegelegt. ${ }^{523}$ Die Nebenfolgen von Regelungen können gravierender sein als der durch das Gesetz anvisierte Zweck. So wies GRUR in der Stellungnahme zum RefE darauf hin, dass die grundsätzliche Vergütungsverpflichtung bei Schranken wie 60a und 60c dazu führen würde/könnte/müsste, dass die

521 Vgl. die Stellungnahme des Hochschullehrerbunds (hlb) zum RefE des UrhWissG: „Konsequenz wäre ein Verzicht auf eine Quantifizierung, etwa bei §60a UrhG-E. Stattdessen könnte als Kriterium dienen, was in der Wissenschaft und in den Vorlesungen zur Veranschaulichung „benötigt“ wird bzw. was „erforderlich“ ist.“ - https://bit.ly/2U2TGJF.

522 Vergriffene Werke (definiert für Werke, die vor dem 1.1.1966 erschienen und die im Buchhandel vergriffen sind) dürfen von Bibliotheken, die diese Werke analog in ihren Beständen haben, im Rahmen von digitalen Bibliotheken für nicht-kommerzielle Zwecke über eine von der VG WORT vergebene Nutzungslizenz digitalisiert und öffentlich zugänglich gemacht werden (geregelt u. a. durch den Rahmenvertrag zur Nutzung von vergriffenen Werken in Büchern - https://bit.ly/ $2 \mathrm{~m} 5 \mathrm{tp0} 5$.

523 Auch in EU-RL-2019, Art. 5, welcher auf cross-border teaching abzielt, ist von Beschränkungen des Umfangs der Nutzung nicht die Rede, selbstverständlich auch nicht für die auf TDM bezogenen Artikel 3 und 4. 
privilegierten Einrichtungen Nutzungsvergütungen zum einen auch für Werke zahlen müssen, die ihre eigenen Mitarbeiter erstellt haben und zum anderen auch für die nach Open Access freigestellten Werken. Das wäre so lange der Fall wie der „Entwurf nicht die Möglichkeit eines Verzichts auf Vergütungsansprüche für den Bereich der Open-Access-Dienste vorsieht." 524 Von sich aus können Urheber nicht im Voraus auf Vergütungsansprüche verzichten.

(15) Gänzlich unakzeptabel ist das Einknicken der Politik gegenüber dem Ansinnen der Presseverleger (initiiert durch intensives Lobbying über ganzseitige Anzeigen der Herausgeber und Geschäftsführer der FAZ ${ }^{525}$ ), Zeitungsartikel aus der Privilegierung herauszunehmen. Diese Forderung war im gesamten Prozess der Entstehung von UrhWissG nie ein Thema, zumal diese Erlaubnis schon seit 2003 durch $\S 52 a$ (der jetzt abgeschafft wird) geregelt war, ohne dass dagegen je ein Protest von Seiten der Medien erhoben wurde oder negative Folgen dieser Regelung bekannt wurden. Forscher (und zunächst auch Lehrende, s. unten) dürfen somit durchaus wissenschaftlich relevanten Zeitungsartikel nicht mehr genehmigungsfrei nutzen, sondern müssen sich Lizenzen dafür bei den Verlagen besorgen.

Nutzungen von ganzen Zeitungsartikeln vor allem für Forschung werden in den nächsten Jahren nur über Einzelkäufe bei den Zeitungsarchiven, Lizenzvereinbarungen oder - als realistische Lösung - über neue Gesamtverträge möglich sein. Letzteres ist für Lehre und Lernen auch schon geschehen. Ende 2018 wurde der Gesamtvertrag „Vervielfältigungen an Schulen“ zwischen KMK und VG WORT (auch für VG Bild-Kunst) abgeschlossen: ${ }^{526}$

Ziel der Vereinbarung ist es, analoge und digitale Vervielfältigungen und einzelne weitere Nutzungen von urheberrechtlich geschützten Schriftwerken, Abbildungen sowie grafischen Aufzeichnungen von Werken der Musik an Schulen auf gesetzlicher oder vertraglicher Grundlage zu ermöglichen sowie hierfür eine angemessene Vergütung festzulegen. (Präambel) ${ }^{527}$

524 GRUR schlägt daher für $\S 60$ g einen neuen Absatz 3 vor: „Eine Vereinbarung, durch welche der Urheber unentgeltlich ein einfaches Nutzungsrecht für jedermann einräumt, geht abweichend von Absatz 1 der gesetzlichen Erlaubnis vor. Dasselbe gilt, soweit der Urheber seinem Dienst- oder Arbeitgeber Rechte eingeräumt hat.“ - https://bit.ly/33QhMfn;

525 Vgl. dazu (Steinhauer 2017) Die Urheberrechtsmärchen der FAZ - https://bit.ly/2RXcH17.

526 Gesamtvertrag 2018 Schulen - https://bit.ly/2MzmZCR.

527 Was ist jetzt durch den Gesamtvertrag erlaubt? „Die Rechtsinhaber gewähren für Lehrkräfte an Schulen, soweit nicht bereits nach $\S 60$ a Abs. 1 UrhG gesetzlich erlaubt, das Recht, nach §2 hergestellte digitale Vervielfältigungen für ihren eigenen Unterrichtsgebrauch zu nutzen, indem sie diese Vervielfältigungen digital per E-Mail an ihre Schüler für den Unterrichtsgebrauch (einschließlich der Unterrichtsvor- und -nachbereitung) weitergeben, ausdrucken und die Ausdrucke ggf. 
Mit diesem Gesamtvertrag deutet sich an, dass der Vorrang von Schrankenregelungen gegenüber Lizenzvereinbarungen (entsprechend §60g) wieder in Frage gestellt wird (vgl. 13.7.2 zur Lizenzierungsplattform). Das kann durchaus auch dazu führen, dass, wie das Beispiel der Nutzung von Zeitungsartikeln zeigt, zu einschränkenden Regelungen im UrhG durch Verträge quasi entschärft werden. Wie die 2019 auf $\S 60 \mathrm{e}$ bezogenen Verträge zeigen (vgl. 13.2.5), können sie aber auch dazu führen, dass die von der Wissenschaft 2017 gegen großen Widerstand der Verlage „erkämpften“ und in $\S 60$ festgeschriebenen Vergütungsformen über Pauschalregelungen (vgl. 13.2.8) wieder teilweise wieder relativiert werden.

\subsection{Ist das UrhWissG ein dauerhafter Ersatz für eine ABWS?}

Dauerhaft schon gar nicht, da auch das UrhWissG zunächst nur als befristet gilt. Aber die Frage muss im politischen Zusammenhang gesehen werden: Kann das UrhWissG als Einlösung der Zusage der Bundesregierung der Großen Koalition in ihrem Koalitionsvertrag von 2013 bewertet werden, eine ABWS in das Gesetz einzuführen? Wir können nicht anders, als diese Frage zu verneinen. Angesichts der vielen im vorigen Abschnitt angesprochenen Defizite muss sogar in Frage gestellt werden, ob das UrhWissG tatsächlich ein wichtiger Schritt in die richtige Richtung ist. Aber wir wollen es uns mit der bewertenden Antwort nicht ganz so einfach machen. Zum einen hat es für die Bundesregierung keineswegs nur die eine Konzeption als Vorgabe für eine ABWS gegeben (vgl. die Abschnitte 12.1.1-12.1.6). Zum anderen sagt die Formulierung in einem Koalitionsvertrag, wie auch so gut alle anderen Stellungnahmen und Forderungen aus der Politik für eine ABWS, so gut wie nichts darüber aus, wie konkret diese ABWS ausgestaltet werden soll. In der Vereinbarung stand lediglich, dass die Koalition mit der ABWS den ,wichtigen Belangen“ von Bildung und Wissenschaft „stärker Rechnung tragen“ möchte. Allerdings hätte der Gesetzgeber einfach auf die Formulierung in Art. 5 Abs. 3 lit. a (Text in FN 188) zurückgreifen können. Das war nach (Raue 2019) schon eine ABWS (vgl. FN 191).

Der Komparativ „stärker“ legt nahe - und darüber waren sich so gut wie alle einig, die aus der Sicht der Wissenschaft und der Politik Stellungnahmen zur ABWS

an die Schüler verteilen, für ihre Schüler über PCs, Whiteboards und/oder Beamer wiedergeben und im jeweils erforderlichen Umfang abspeichern, wobei auch ein Abspeichern auf mehreren Speichermedien der Lehrkraft gestattet wird (PC, Whiteboard, iPad, Laptop, etc.), jedoch Zugriffe Dritter durch effektive Schutzmaßnahmen verhindert werden müssen (Passwort etc.). “(§3) Bezüglich des Umfangs ist erlaubt: „Noten im Umfang von maximal 6 Seiten; -Schriftwerke, mit Ausnahme von Unterrichtswerken, im Umfang von maximal 20 Seiten; Pressebeiträge; Bilder, Fotos und sonstige Abbildungen; vergriffene Werke“ (§4) (FN 527). 
abgegeben hatten -, dass die bisherigen Schrankenregelungen diesen „Belangen“ nicht ausreichend gerecht geworden sind. Unbestreitbar trägt das UrhWissG diesen Belangen jetzt weitergehend Rechnung trägt, als es durch die bisherigen Schranken geschehen war. Das scheint für die Politik bei der Beschlussfassung in Regierung und Bundestag schon der ausreichende Grund gewesen zu sein, UrhWissG als ABWS anzusehen. Opposition und Bundesrat (und damit die Länder als für Bildung und Kultur zuständige Verfassungsorgane) sahen das allerdings anders. Aber das sind nicht die für die Gesetzgebung im Urheberrecht entscheidenden politischen Gruppierungen bzw. Instanzen.

Fazit. Man kann es drehen und wenden, wie man will: Der Anspruch einer ABWS bzw. einer ABWK wurde durch das UrhWissG nicht eingelöst. Dieser Anspruch zielt auf eine einheitliche einfache allgemeine umfassende Regelung zugunsten der Interessen von Bildung und Wissenschaft, die sich ausschließlich an dem Zweck der Nutzung orientiert. Werden die internationalen und vor allem die EU-Vorgaben, ebenso alle, obschon zum Teil ziemlich weit zurückliegenden Urteile/Entscheidungen der obersten deutschen Gerichte (BGH und BVerfG) als wörtlich zu nehmende und nicht hintergehbare Vorgaben angesehen, dann besteht allerdings wenig Spielraum für eine ABWS/K. Aber die wortwörtliche Auslegung bestehender Vorgaben oder auch nur das Herausarbeiten dessen, was Gesetzgeber und Richter vielleicht gemeint haben, wird dem Auftrag beider nicht gerecht, das Recht in Einklang mit dem zu bringen, was sich in der Öffentlichkeit als moralisches Bewusstsein für den Umgang mit Wissen und Information entwickelt hat. Aber auch im Urheberrecht kann möglich gemacht werden, was lange Zeit als unmöglich galt. Erforderlich, möglich, nicht unmöglich sollte es sein, das in das Recht umzusetzen, was sich in der Gegenwart über Leitideen wie die der Nutzungsfreiheit herausbildet hat. ${ }^{528}$ Dafür hat der Gesetzgeber jetzt erneut einige Jahre Zeit. Das UrhWissG ist zwar seit 2018 geltendes Recht, aber es ist erst einmal auf 5 Jahre befristet und wird inzwischen teilweise durch die EU-Vorgaben in DSM-RL korrigiert. Auf der Grundlage einer Evaluierung, ein Jahr vor Ablauf der Frist, soll entschieden werden, wie es damit weitergeht - vielleicht doch zu realisieren, was bislang als unmöglich galt. Dazu einige Vorschläge im nächsten Abschnitt.

528 Thomas Hartmann hat die Annäherung des Rechts an Leitideen als Fortschreibung von bewährten und in der Praxis bewährten „Leitlinien“ beschrieben: „Nach einer Phase der Bewährung und steigenden Akzeptanz in der Wissenschaftseinrichtung liegt es häufig nahe, Empfehlungen und Leitlinien einer Open Access Policy zu rechtlich verbindlichen Standards fortzuschreiben. Eine in der Publikationspraxis gereifte Open Access Policy kann damit als Vorläufer einer rechtsverbindlichen Satzung mit Open Access-Publikationspflichten dienen“ (Hartmann 2017) Zwang zum Open Access-Publizieren? 


\section{6 Änderungs-/Verbesserungsbedarf im aktuellen Wissenschaftsurheberrecht}

Unabhängig von dem Ziel der Allgemeinen Bildungs- und Wissenschaftsklausel besteht weiterer konkreter Verbesserungsbedarf im Wissenschaftsurheberrecht, der bei der in vier Jahren anstehenden Evaluierung ebenfalls berücksichtigt werden sollte. Auch durch die Verabschiedung der EU-DSM-RL von 4/2019 muss das deutsche Urheberrecht bis spätestens 6/2021 an verschiedener Stelle angepasst werden. ${ }^{529}$

Die direkt durch das UrhWissG entstandenen bzw. nicht gelösten Probleme sind in der Liste in 13.4 aufgeführt. Probleme bestehen aber zum einen bei einigen früher im Gesetz festgeschrieben Regelungen und zum anderen bei im UrhWissG nicht angegangenen Themen,

(1) Für Bibliotheken ist bislang für E-Books keine Regelung vorgesehen, die dem Rechnung trägt, dass sie im öffentlichen Interesse E-Books sowohl erwerben als auch ausleihen dürfen. Praktikable, auch den Interessen der Verlage entgegenkommende Vorschläge von Seiten der Bibliotheken liegen vor. ${ }^{530}$

529 Für Bildung und Wissenschaft einschlägig sind z. B. die folgenden Artikel aus EU-DSM-RL: Art. 3 Text und Data Mining zum Zwecke der wissenschaftlichen Forschung (eventuell auch Art. 4 Text und Data Mining)

Art. 5 Nutzung von Werken und sonstigen Schutzgegenständen für digitale und grenzüberschreitende Unterrichts- und Lehrtätigkeiten

Art. 6 Erhalt des Kulturerbes (Art. 7 in Verbindung mit Art. 3, 5, 6)

Art. 8 Nutzung von vergriffenen Werken und sonstigen Schutzgegenständen durch Einrichtungen des Kulturerbes (Art. 9-11 mit Bezug auf Art. 6)

Art. 12 Kollektive Lizenzvergabe mit erweiterter Wirkung

Art. 15 Schutz von Presseveröffentlichungen im Hinblick auf die Online-Nutzung(zusammen mit Art. 16)

Art. 17 Nutzung geschützter Inhalte durch Diensteanbieter für das Teilen von Online-Inhalten. Die Bundesregierung hat Januar 2020 in einem ersten Vorschlag auf diese Vorgaben der EU reagiert (vgl. FN 480), vor allem mit Blick auf $\S 60$ a und 60d sowie auf das Leistungsschutzrecht und die Verlegerbeteiligung an der Vergütung. Das wurde bei der Diskussion hier schon berücksichtigt, z. B. in 11.4 und 8.7).

530 Vgl. dbv: E-Books in Bibliotheken - https://bit.ly/30w4h2j]; vgl. auch den entsprechenden Antrag der LINKE: Verleihbarkeit digitaler Medien entsprechend analoger Werke in Öffentlichen Bibliotheken sicherstellen. Drucksache 18/5405 vom 1.7.2015 - https://bit.ly/2ZqPHHY. Der Vorschlag wurde vom Bundestag nicht angenommen.

Das Aktionsbündnis (entsprechend der Einschätzung des stellvertretenden Sprechers Oliver Hinte) hatte Ende Januar in einer ersten, an das BMJV übermittelten Stellungnahme zu dem Diskussionsvorschlag der Bundesregierung zur Umsetzung von EU DSM-RL (https://bit.ly/39rcaLT) auch die Anwendung des E-Ausleihe-Urteils des EuGH (Rechtssache C-174/15) über eine Erweiterung 
(2) Dem öffentlichen Interesse an der elektronischen Verfügbarkeit der Materialien in Museen und Archiven sollte zeitgemäß durch eine neue Schranke (als Ersatz für §60f) Rechnung getragen werden (vgl. die Punkte 10 und 11 in der Liste in 13.4)..$^{531}$

(3) Die Regelungen in den 95er-Paragraphen zum Schutz von technischen Schutzmaßnahmen (DRM) bestehen weiter. Dieser „alte Zopf“ aus der EU-Richtlinie von 2001, dem der Gesetzgeber in Deutschland 2003 meinte folgen zu müssen, ist nicht länger haltbar. Technische Schutzmaßnahmen (DRM) sollten in Bildung und Wissenschaft grundsätzlich nicht zum Einsatz kommen. Die Anstrengungen zur Langzeitarchivierung, die der Sicherung des kulturellen Erbes der Bibliotheken und Archive dienen, aber auch neue Nutzungshandlungen wie TDM ermöglichen, sollten nicht durch DRM behindert werden.

(4) In der 2014 gültig gewordenen Regelungen für ein Zweitverwertungsrecht in $\S 38 \mathrm{Abs.} 4 \mathrm{UrhG}$ ist bislang nicht abschließend geklärt, ob der in der Begründung des Zweitverwertungsrechts eindeutig erwähnte Ausschluss der grundfinanzierten Hochschulforschung für dieses Recht verbindlich ist oder nicht. Neben der Korrektur an anderen in 11.3.4 aufgeführten Unzulänglichkeiten des Zweitverwertungsrechts ist jetzt eine schrittweise zu realisierende Öffnungsklausel für größere Werke wie Monographien und wissenschaftliche Lehrbücher in das Zweitverwertungsrecht erforderlich und zeitgemäß.

(5) Die Nutzung von verwaisten Werken sollte intensiviert werden, vor allem dadurch, dass die „sorgfältige Suche“ zugunsten einer zeitlich befristeten öffentlichen Auslegung einer beabsichtigen Nutzung eines verwaisten Werks abgeschafft wird.

(6) Eine Wiederaufnahme des Leistungsschutzrechts für Presseverleger, das sich als überflüssig und nutzlos erwiesen hat, sollte vermieden werden. Weder haben sich die Erwartungen der Verleger und schon gar nicht der Journalisten selbst an zusätzlichen Einnahmen erfüllt. Falls Suchmaschinenhersteller in Zukunft zur Vermeidung von Lizenzansprüchen der Verleger nicht mehr auf Presseerzeugnisse referenzieren sollten, wird es für Bildung und Wissenschaft

von $§ 27$ Abs. 2 gefordert. Darin hatte der EuGH entschieden, dass eine gesetzliche Regelung der elektronischen Bibliotheksausleihe im nationalen Recht möglich ist und dass sich die E-Ausleihe nicht grundsätzlich von der Leihe analoger Werke unterscheidet.

531 Leider hat es die Bundesregierung versäumt bzw. die EU nicht davon überzeugen können, dass Museen und Archive nicht nur einen Bewahrungs- und Bestandssicherungsauftrag haben, sondern auch die öffentliche Zugänglichmachung ermöglichen sollten. Die EU erlaubt in „Artikel 6 Erhaltung des Kulturerbes” in der neuen Richtlinie von 2019 weiter nur das Vervielfältigen von Beständen in diesen Einrichtungen „Zwecke der Erhaltung dieser Werke oder sonstigen Schutzgegenstände in dem für diese Erhaltung notwendigen Umfang“. 
sehr schwierig, den Überblick über forschungsrelevante Presseartikel zu behalten. Allerdings wird der deutsche Gesetzgeber kaum die Vorgabe der EU von Art. 13 in DSM-RL 2019 ignorieren können. Der umfassenden Kritik an dem Leistungsschutzrecht für Presserverleger aus Wissenschaft, Zivilgesellschaft, aber auch aus weiten Kreisen der Politik selbst wird nicht Rechnung getragen.

Die UrhWissG-Folgediskussion wird bislang stark vom pro und contra einer Lizenzierungsplattform bzw. dem Thema der Lizenzierung allgemein bestimmt. Damit ist eine erneute Diskussion um die Priorität von Schrankenregelungen gegenüber Lizenzierungsvereinbarungen zu erwarten. Wir gehen daher am Ende der Auseinandersetzungen mit den Urheberrechtsreformen seit 2003 auf diese Problematik ein. Der Ausgang wird darüber entscheiden, ob das Urheberrecht weiter ein Regulierungsmittel im Interesse der Öffentlichkeit sein soll oder ob die Gestaltung des Umgangs mit Wissen und Information weitgehend den kommerziellen Interessen überlassen wird - in diesem Fall über Lizenzierungsverfahren.

\subsection{Lizenzen vs. Urheberrechtsschranken}

Lizenzen sind aus der digitalen Publikationswelt nicht wegzudenken. Sie können auch für Bildung und Wissenschaft ein nützliches Instrument sein, a) wenn die Nutzungsbedingungen offengelegt werden; b) wenn die Nutzung nach offenen Prinzipien geregelt wird, z. B. indem die lizenzierten Objekte unter eine Creative-Commons-Lizenz gestellt werden können; c) wenn Lizenzen die im Urheberrecht vorgesehenen Schrankenbestimmungen anerkennen, diese also nicht durch vertragliche Vereinbarungen außer Kraft gesetzt werden können; d) wenn durch schrankenbasierte Nutzungen keine neuen Vergütungsansprüche entstehen. Andernfalls, so IFLA, behindern Lizenzen wissenschaftliche Kreativität und wirtschaftliche Innovationskraft:

Replacing copyright law entirely with licensing restricts access to, and reuse of digital knowledge, and is hampering research and creativity.

It is vital that governments ensure that education, research, science and the public interest are protected from the information monopolies created by licences. Otherwise, access to education and research, will be determined by the commercial interests of private companies with little or no concern for the wider needs of society. ${ }^{532}$

532 (IFLA 2015) IFLA critiques licensing solutions at the European Parliament. 


\subsubsection{Aushebelung von Schrankenregelungen durch Lizenzangebote}

Um das Verhältnis von Lizenzierungsregelungen vs. Schrankenregelungen wurde schon seit langem gestritten. Bei der Reform des Urheberrechts 2003 war nur bei den $\S \S 52 \mathrm{~b}$ und 53a, nicht bei §52a eine Einschränkung der Schrankenbestimmungen durch vertragliche Vereinbarungen/Lizenzen vorgesehen. ${ }^{533}$ Daraus schloss der BGH aber nicht, dass bei §52a eine solche Beschränkung nicht möglich sei. Nach seiner Ansicht, nach seiner Auslegung der Vorgaben von InfoSoc 2001 Art. 5 Abs. 3, lit. a (ergänzt durch EG 45 der Richtlinie) spräche nichts dagegen, dass

die Inanspruchnahme der Schrankenregelung (unter anderem) dann nicht geboten ist, wenn ein angemessenes Lizenzangebot vorliegt. Ein Vorrang angemessener Lizenzangebote ermöglicht es dem Rechtsinhaber auch nicht, einseitig Bedingungen festzulegen und die Schranke des §52a UrhG auszuhebeln. Das Angebot des Rechtsinhabers ist nur vorrangig, wenn die Bedingungen angemessen sind.

Als Bedingung für eine angemessene Lizenzierung empfahl der BGH für §52a, dass „das Lizenzangebot unschwer aufzufinden ist und die Verfügbarkeit des Werkes oder der Werkteile schnell und unproblematisch gewährleistet ist.“ Bei §52b war er sich insgesamt und auch bei Vorliegen von Verlagsangeboten nicht ganz so sicher und hatte daher den EuGH um Überprüfung gebeten. In dessen Urteil vom 11.9.2014 verneinte der EuGH ausdrücklich, dass schon ein Verlagsangebot, auch wenn es den Eindruck macht, zu angemessenen Bedingungen erstellt zu sein, die durch das Recht vorgegebenen Schrankenbestimmungen für die Nutzung unwirksam macht. Also Vorrang der Schrankenregelung. Dieses Urteil, zunächst ja nur auf den inzwischen aufgehobenen $\S 52 \mathrm{~b}$ bezogen, hatte Auswirkungen auf den allgemeinen, höchst kontrovers diskutierten Streit „Lizenz vs. Schranken“. Der Gesetzgeber hatte sich bei der UrhWissG-Reform in $\S 60 \mathrm{~g}$ den vorsichtig gesagt konservativen Auslegungen ${ }^{534}$ nicht angeschlossen, sondern ist (mit Einschränkungen) eher

533 §52b-alt, Satz 1: „,soweit dem keine vertraglichen Regelungen entgegenstehen“) und 53a-alt Abs. 1 Satz 3: ,wenn der Zugang [...] nicht offensichtlich [...] mittels einer vertraglichen Vereinbarung zu angemessenen Bedingungen ermöglicht wird“.

534 (de la Durantaye 2014a) interpretierte in den Erläuterungen zu §XX unter B 3, dass aus dem „geboten“ eindeutig der „Vorrang von Verlagsangeboten“ abzuleiten sei: „Die Schranke [gemeint ist $\S X X]$ soll nicht in jedem Fall privatautonomen Lösungen vorgehen.“ (231) Begründet wurde die Priorität von Lizenzangeboten gegenüber Schrankenrechten mit maximal starker, rechtsdogmatischer Munition (Verfassungsrecht, Völkerrecht, Drei-Stufen-Test und Europarecht/InfoSoc-Richtlinie): „Dies [der Vorrang des Lizenzangebots] entspricht verfassungs- und völkerrechtlichen Vorgaben. Aufgrund der Institutsgarantie des Eigentums nach Art. 14 Abs. 1 GG1636 dürfte der Ersatz von Ausschließlichkeitsrechten durch Vergütungsansprüche im Kerngeschäftsfeld des Rechtsinhabers 
dem EuGH-Urteil gefolgt: Die Priorität von Nutzungsbedingungen in gesetzlichen Schrankenregelungen gegenüber Lizenzierungsvereinbarungen wurde ins Gesetz festgeschrieben ( $\$ 60 \mathrm{~g}$ Abs. 1). ${ }^{535}$ Allerdings - und dies schränkt die prinzipielle Priorität ein - gilt diese Priorität zum einen nur, wenn Lizenzierungsvereinbarungen die gesetzlich erlaubten Regelungen „beschränken oder untersagen“. Zum anderen wird diese Priorität bei zentralen Nutzungshandlungen ausgesetzt, wie „die Zugänglichmachung an Terminals [in Bibliotheken - RK] nach §60e Absatz 4 und $\S 60 f$ Absatz 1 oder der Versand von Vervielfältigungen auf Einzelbestellung nach $\S 60$ e Absatz 5“.

\subsubsection{Lizenzen über Lizenzierungsplattformen}

Die Diskussion um Lizenzierung ist auch mit dem UrhWissG nicht abgeschlossen, ganz im Gegenteil. Vor allem die Verlagswirtschaft wollte sich keineswegs mit der Priorisierung der Schrankenregelungen abfinden: „Vorrang von Lizenzangeboten zu angemessenen Bedingungen gegenüber Schrankennutzungen“ - so die zentrale Forderung des Börsenvereins des Deutschen Buchhandels auch nach dem UrhWissG. ${ }^{536}$ Die Bundesregierung im Koalitionsvertrag 2018 (CDU/CSU und SPD) hatte sich verpflichtet auszuloten,

wie der Zugang zu wissenschaftlichen Publikationen im Interesse aller Beteiligten - der Autorinnen und Autoren, der vielfältigen deutschen Verlagslandschaft und der nutzenden Wissenschaft - über eine Lizenzierungsplattform verbessert werden kann. ${ }^{537}$

Das BMJV hatte rasch den Auftrag der Bundesregierung umgesetzt und für den 13./14. September 2018 die beteiligten Akteure zu einer Auftaktveranstaltung „Dia-

nicht zulässig sein. Mit der zweiten und dritten Stufe des Drei-Stufen-Tests [...] wäre es ebenfalls unvereinbar, wenn Nutzungen nach $\S$ XX stets zulässig wären, obwohl Rechtsinhaber sie als zugeschnittene Leistungen in ihrem Kerngeschäft anbieten.“ (231)

535 Laut IFLA (vgl. FN 532) schützen auch andere EU-Länder ihre urheberechtlichen Normen davor, durch vertragliche Lizenzvereinbarungen ausgesetzt zu werden, z. B. UK, Irland, Portugal and Belgien. Auf EU-Ebene (ebenso nach IFLA): „The European Software Directive and Database Directive also protects exceptions from contractual override.”

536 Christian Sprang, Justiziar des Börsenvereins, in einem Vortrag auf der Bielefelder Auftaktveranstaltung „Dialog Lizenzierungsplattform“ im September 2018. Anders z. B. Schmücker auf der gleichen Veranstaltung: „Schranken des Urheberrechts dürfen deshalb nicht durch eine Lizenzierungsplattform ausgehebelt werden“ - https://bit.ly/2QPeWB2.

537 Koalitionsvertrag 2008 - https://bit.ly/35XgfE2. 
log Lizenzierungsplattform“ in das ZIF/Bielefeld eingeladen. ${ }^{538}$ Nach Bielefeld haben dazu weitere Veranstaltungen stattgefunden. ${ }^{539}$ Zum Zeitpunkt des Abschlusses dieses Textes zeichnet sich noch kein Konsens über Konzeption, Verfahren, Institutionalisierung, Abrechnungsformen, Finanzierung etc. ab, ebenso nicht, wie das Zusammenspiel zwischen Schrankenregelungen und Lizenzvereinbarungen geregelt werden kann. Allerdings besteht durch die Verabschiedung von EU-DSMRL2019 die Verpflichtung der Mitgliedsländer, Art. 12 Kollektive Lizenzvergabe mit erweiterter Wirkung bis spätestens Juni 2021 umzusetzen (vgl. Axhamn 2019) (in Deutschland vorgesehen über $\S 61 \mathrm{f}-\mathrm{https} / / /$ bit.ly/2WFOmhM). Die EU ist damit weitgehend den Vorstellungen gefolgt, die auf den Erfahrungen bezüglich der "Extended Collective Licences" (ECL) beruhen, vor allem in den nordischen Ländern (Riis/Schobsbo 2010), aber auch in Ländern wie den USA (Gervais 2011), Kanada (Gervais 2003) oder China (Jiang/Gervais 2012). Auf diese Modelle bezieht sich auch in Deutschland der Börsenverein des Deutschen Buchhandels, der das Konzept einer Lizenzierungsplattform intensiv verfolgt (s. oben). ${ }^{540}$ Es wird aber in der Literatur durchaus davor gewarnt, dieses Modell auf andere Länder direkt zu übertragen:

If one, therefore, would seek to transplant these models one should be very careful to include into the design of the systems not just the rules themselves but also the broader background of right holders' organization, competition law control etc. (Riis/Schovsbo 2010, 25; vgl. Weber ${ }^{541}$ 2017)

Auch in der EU wird über verschiedene Erwägungsgründe (EG 44ff) darauf hingewiesen, dass den Besonderheiten des jeweiligen Mitgliedlandes Rücksicht genommen werden sollte. Es fällt dabei auf, das weder im Text von Art. 12 noch in den zahlreichen EG von Wissenschaft die Rede ist. Auch die Literatur zu den ECL in den nordischen Ländern bezieht sich überwiegend auf Literatur und Kunst. Im Fol-

538 Auf der Website des BMJV werden i. d. R. die Materialien der Veranstaltungen nachgewiesen (vgl. FN 536).

539 24. Oktober 2018 Regensburg (aus der Sicht experimenteller Forschung); 30. Januar 2019 Weinheim (auf Einladung des Börsenvereins des Deutschen Buchhandels e. V., mit Beteiligung der Verlage Beltz und Wiley-VCH); 28. Februar 2019 Osnabrück (auf Einladung des Zentrum virtUOS der Universität Osnabrück); 15. Mai 2019 Göttingen (zum Verwalten von Daten aus Forschungseinrichtungen, Bibliotheken, Verlagen, Museen und Archiven, auf Einladung von Universitäts-, Max-Planck- und Bibliotheksrechenzentren); 27. Mai 2019 Ilmenau (Auswirkungen der Einführung einer Lizenzierungsplattform, auf Einladung des dbv); 12. Juni 2019 Berlin (Veranstaltung des Wikimedia Deutschland e. V.). Vermutlich werden weitere Veranstaltungen stattfinden.

540 Auch die initiative urheberrecht begrüßt die durch die ECL möglich werdende Lizenz „aus einer Hand“ - https://bit.ly/2ngGrZV, I,4. Jedoch wird hier eher Bezug allgemein auf das kulturelle Erbe genommen, nicht explizit auf Bildung und Wissenschaft.

541 (Weber 2017) Die urheberrechtliche Zwangslizenz. 
genden werden, unabhängig von den Vorgaben von Art. 12 der EU-DSM-RL2019, Anforderungen an eine Lizenzierungsplattform zur Diskussion gestellt, die auf Bildung und Wissenschaft bezogen sind und sich damit nicht an einem exklusiven kommerziellen Verwertungsinteresse orientieren (vgl. FN 536):

(1) Oberste Priorität für Lizenzierungsplattformen ist, die Nutzungsbedingungen für Bildung und Wissenschaft zu verbessern.

(2) Der wesentliche Zweck von Lizenzierungsplattformen sollte es sein, den Vermittlungsorganisationen (z. B. Bibliotheken) die Bereitstellung publizierter Objekte für ihre Nutzer zu erleichtern.

(3) Durch Lizenzierungsplattformen sollte die Regelung des §60g UrhG nicht außer Kraft gesetzt werden, die besagt, dass gesetzliche (Schranken-)Regelungen i. d. R. vertraglichen Regelungen vorgehen.

(4) Durch Lizenzierungsplattformen sollen individuelle schrankenbasierte Nutzung nicht erfasst werden.

(5) Eine effiziente öffentliche/staatliche Aufsicht über eine entsprechende Einrichtung ist erforderlich. Nur so kann die gewichtete Balance zwischen den kommerziellen Interessen der Verwertung und dem prioritären öffentlichen Interesse an Gemeinfreiheit der Nutzung von publizierter Information in Bildung und Wissenschaft erreicht werden. Lizenzierungsplattformen sollten keine Service-Einrichtung der Verlagswirtschaft sein. ${ }^{542}$

(6) Entsprechend der in dieser Arbeit aufgestellten Forderung, sollten der Verlagswirtschaft über Lizenzen nur einfache Nutzungsrechte zugesichert bekommen.

(7) Die Öffentlichkeit (Bund und/oder Länder) stellt der Lizenzierungsplattform über einen Globalbetrag die Mittel für die Nutzung der in Deutschland verlegten Publikationen bereit und sorgt unter öffentlicher/staatlicher Aufsicht für die Verteilung an die entsprechenden kommerziellen Rechtsinhaber. ${ }^{543}$

(8) Über Lizenzierungsplattformen sollte eine Symmetrie auch mit Blick auf Lizenzierung erreicht werden, welche derzeit nicht gegeben ist. Mit Symmetrie ist gemeint, dass nicht nur (wie bislang) die Bildung und Wissenschaft zuarbeitenden Vermittlungsorganisationen Lizenzgebühren an die Vertreter der Verlagswirtschaft zahlen müssen, sondern dass die Vertreter der Verlags-

542 So auch Schmücker auf der Bielefelder Tagung: „Eine Lizenzierungsplattform bedarf deshalb eines Trägers, der (vielleicht vergleichbar einer Regulierungsbehörde) von Rechtsinhabern und Wissenschaftseinrichtungen unabhängig und fair zwischen durch die unentgeltliche Nutzung öffentlicher Einrichtungen urheber- und verwerterseitig ersparten Aufwendungen und verwerterseitig geleistetem Veredelungsaufwand abzuwägen vermag“ (vgl. über FN 536).

543 Ähnlich Schmücker in einem Gedankenexperiment (vgl. über FN 536). „Der Staat subventioniert indirekt den weltweiten Zugang zu in Deutschland verlegter wissenschaftlicher Literatur.“ Allerdings ist aus dem „,indirekt“ längst ein „direkt“ geworden - so im DEAL-deal (vgl. 14.8). 
wirtschaft an die Institutionen der Urheber Lizenzgebühren als Voraussetzung für ihre kommerzielle Verwertung entrichten müssen. Eine solche spezielle Symmetrieforderung ist bislang so gut wie gar nicht in die Diskussion einbezogen worden. ${ }^{544}$

(9) Durch Lizenzen sollten keine umfangsbeschränkten Nutzungen festgelegt werden (wie sie derzeit in Schrankenregelungen meistens vorkommen), d. h. die Lizenzen sollten es den Nutzern in Bildung und Wissenschaft überlassen, in welchem Umfang sie die lizenzierten Werke nutzen wollen.

(10) Bei Erwerb einer Lizenz, z. B. durch eine Bibliothek, sollten keine weiteren Vergütungsansprüche der entsprechenden Verlage durch konkrete schrankenbegünstigte Nutzungssituationen entstehen, auch wenn der Umfang der konkreten Nutzungen über die derzeit bestehenden Schrankenregelungen hinaus geht.

(11) Durch Lizenzierungsplattformen sollte die gemäß §60h UrhG regelmäßig vorgesehene Pauschalvergütung nicht durch Aufsummierung von Einzelabrechnungen ersetzt werden.

(12) Den Bildung und Wissenschaft zuarbeitenden Vermittlungsorganisationen darf der Erwerb von Lizenzen für publizierte Wissensobjekte (aktuell für das Ausleihen von E-Books) nicht verweigert werden.

(13) Lizenzen dürfen von den sie in Anspruch nehmenden Einrichtungen für alle (im UrhG definierten) Verwertungsrechte genutzt werden. Nicht nur Bibliotheken, sondern auch Museen und Archive dürfen somit das Recht der öffentlichen Zugänglichmachung umfassend (anders im UrhWissG geregelt) in Anspruch nehmen.

(14) Lizenzierungsangebote der Verlagswirtschaft enthalten i.d. R. verschlüsselte Verfahren des DRM, die im jetzigen Urheberrecht über die $\S \S 95 a-d$ geschützt sind. Falls es bei diesen bleiben sollte, müssten bei der Lizenzierungsplattform Schlüssel zur Entschlüsselung solcher Verfahren von der Verlagswirtschaft hinterlegt werden, damit es im Streitfall über zulässige oder nicht zulässige Nutzungsregelungen nicht zu unbillig langen gerichtlichen Auseinandersetzungen kommen muss.

544 Die bislang übliche Praxis, nämlich dass die Urheber die direkten Vertragspartner für den Prozess der Übertragung der Übertragung der den Urhebern zustehenden Verwertungsrechte als Nutzungsrechte an die kommerziellen Verwerter sind, trägt wesentlich zum Ungleichgewicht bei der Vertragsausgestaltung zwischen Urheber und Verwerter bei. Aber vor allem berücksichtigt diese Praxis, die im Übrigen oft genug zu keinerlei Vergütung an die Urheber führt, in keiner Weise die erheblichen Vorleistungen, die die Öffentlichkeit für die Erstellung von Wissensobjekten erbringt. Wir haben schon bei der Diskussion um das Zweitverwertungsrecht für die Sicherung institutioneller Rechte im Urhebervertragsrecht plädiert. 
(15) Lizenzen dürfen erweiterte (bzw. bislang unbekannte) Nutzungsformen wie TDM bzw. allgemein: Data Analysis oder Verfahren der Textanalyse nicht ausschließen.

(16) Lizenzen dürfen nicht vorsehen, dass die entsprechenden Materialien für eine externe Nutzung über VPN nicht genutzt werden können.

Die Diskussion Lizenzierung vs. Schranken wird das Urheberrecht noch weiter beschäftigen. Das zeigen die Diskussionen um die Lizenzierungsplattform. Diese werden bis zu der für 2022 vorgesehenen Evaluierung des UrhWissG vermutlich nichts an den Regelungen in §60e ändern. Auch CR-RL2019 gibt dazu zwar keine verbindlichen Vorgaben, aber doch einige Hinweise für den Spielraum der Gesetzgebung:

\begin{abstract}
So könnten Mitgliedstaaten insbesondere beschließen, die Anwendung der Ausnahme oder Beschränkung [für Bildungs-/ausbildungszwecke - RK] vollständig oder teilweise von der Verfügbarkeit geeigneter Lizenzen abhängig zu machen, die mindestens dieselben Nutzungen abdecken wie die im Rahmen der Ausnahme oder Beschränkung genehmigten. Die Mitgliedstaaten sollten dafür Sorge tragen, dass Lizenzen nicht nur die als Ausnahme oder Beschränkung zulässigen Nutzungen, sondern auch möglichst alle übrigen Nutzungen ermöglichen.
\end{abstract}

Die Mitgliedstaaten könnten dieses Verfahren beispielsweise anwenden, um den Lizenzen für Materialien, die vor allem für den Bildungsmarkt gedacht sind, oder Lizenzen für Notenblätter Vorrang einzuräumen. Damit es nicht zu Rechtsunsicherheit oder Verwaltungsaufwand für Bildungseinrichtungen führt, wenn die Anwendung der Ausnahme von der Verfügbarkeit von Lizenzen abhängig gemacht wird, sollten die Mitgliedstaaten, die sich für ein solches Konzept entscheiden, konkrete Maßnahmen ergreifen, um die einfache Verfügbarkeit von Lizenzierungsmodellen zu gewährleisten, die die digitale Nutzung von Werken und sonstigen Schutzgegenständen für Zwecke der Veranschaulichung des Unterrichts ermöglichen, und dafür sorgen, dass diese Lizenzierungsmodelle den Bildungseinrichtungen auch bekannt sind. Solche Lizenzierungsmodelle sollten den Bedürfnissen der Bildungseinrichtungen entsprechen. (EG 23)

Die optionalen Formulierungen erlauben es dem deutschen Gesetzgeber, auch dauerhaft an den jetzigen Präferenzen in $\S 60 \mathrm{~g}$ festzuhalten, aber diese auch über eine Lizenzierungsplattform zu ändern.

Fazit. Lizenzierungsverfahren bzw. die Lizenzierungsplattform werden so wie sie derzeit im Rahmen des „Dialog Lizenzierungsplattform“ diskutiert werden (vgl. FN 538), in erster Linie die Einnahmesituation der kommerziellen Anbieter stabilisieren oder sogar verbessern. Das ist nicht das primäre Interesse von Bildung und Wissenschaft. Fraglich, ob durch Lizenzierungsplattformen das Ziel eines verbesserten Zugangs zu wissenschaftlichen Publikationen erreicht werden kann. 
Die Frage nach dem „ob“ hat sich die Bundesregierung aber nicht gestellt, sondern nur nach dem „wie“(s. das Zitat oben). Eine Fortsetzung der Unterstützung kommerzieller Verwertungsinteressen geht nicht zusammen mit der hier vertretenen und sich immer stärker entwickelnden Leitidee der Nutzungsfreiheit für das öffentlich zugänglich gemachte Wissen. Die ganze Debatte um Lizenzierungsplattform wirkt, wie viele andere Themen im Urheberrecht, besonders stark wie aus der Zeit gefallen. Bildung und Wissenschaft brauchen vom Gesetzgeber die Garantie der Nutzungsfreiheit, nicht die in Lizenzverträgen zwangsläufig vorgesehenen Nutzungseinschränkungen, die zudem die rechtlichen Vorgaben aus den Schrankenregelungen außer Kraft setzen. Lizenzierungsplattformen passen im Übrigen überhaupt nicht zu dem PAR-Ansatz (publish\&read) von DEAL (vgl. 14.8), der allerdings bislang nur auf Zeitschriften Anwendung findet. Insgesamt sollte in Zukunft nur die Erstellung von Informationsobjekten finanziert werden, aber nicht die Nutzung. Das entzöge Lizenzierungsplattformen die Grundlage - im Übrigen auch die für Schrankenregelungen. 